\title{
Ultraviolet optoelectronic devices based on AIGaN alloys grown by molecular beam epitaxy
}

Theodore D. Moustakas, Electrical and Computer Engineering Department, Division of Materials Science and Engineering, Photonics Center, Boston University, Boston, MA 02215, USA

Address all correspondence to Theodore D. Moustakas at tdm@bu.edu

(Received 25 April 2016; accepted 27 July 2016)

\section{Abstract}

This paper reviews progress in ultraviolet (UV) optoelectronic devices based on AlGaN films and their quantum wells (QWs), grown by plasmaassisted molecular beam epitaxy. A growth mode, leading to band-structure potential fluctuations and resulting in AIGaN multiple QWs with internal quantum efficiency as high as $68 \%$, is discussed. Atomic ordering in these alloys, which is different from that observed in traditional III-V alloys, and its effect on device performance is also addressed. Finally, progress in UV-light-emitting diodes, UV lasers, UV detectors, electroabsorption modulators, and distributed Bragg reflectors is presented.

\section{Introduction}

The field of AlGaN-based ultraviolet (UV) optoelectronic devices (primarily emitters and photodetectors), has been an active area of research over the past two decades. ${ }^{[1-5]}$ AlGaN alloys are well suited for such devices because their energy gap can be tuned by changing the alloy composition to cover the entire UV spectral region from 210 to $360 \mathrm{~nm}$. This research is motivated by a plethora of potential industrial and medical applications. Such include, for example, free-space non-line-of-sight communications, water/air/food sterilization, surface disinfection, fluorescence or Raman identification of biological/chemical agents, epoxy curing, counterfeit detection, and various diagnostic and therapeutic medical applications. However, despite intense efforts worldwide, the maximum external quantum efficiency (EQE) of fully packaged AlGaN-based deep UV-light-emitting diodes (LEDs), emitting below $300 \mathrm{~nm}$, is only 1\%-3\%. ${ }^{[5-9]}$ Only recently Shatalov et al. reported a UVLED emitting at $278 \mathrm{~nm}$ with EQE of about $10 \%{ }^{[10]}$ This is to be contrasted with InGaN-based violet-blue LEDs, whose EQE is more than $50 \%$. $^{[11]}$

The EQE is defined as the product of the internal quantum efficiency (IQE), the injection efficiency (IE), and the extraction efficiency (EE). Thus, the poor EQE of the deep UV-LEDs may be the result of poor IQE, IE, EE, or a combination of all three factors. The IQE depends sensitively on extended and point defects, which act as non-radiative recombination centers. The IE depends on the ability to dope the $\mathrm{n}$ - and p-sides of the junction efficiently and to form Ohmic contacts with low contact resistivity on both sides. The EE depends sensitively on the ability to extract the light from the active region of the device to the free space.
The development of AlGaN-based UV lasers is even at an earlier stage of development. Several groups have reported the development of optically pumped deep UV lasers as well as the demonstration of stimulated emission. ${ }^{[12-19]}$ However, the reported shortest wavelength of electrically pumped UV lasers is 336 nm. ${ }^{[20]}$ Significant progress has been made in AlGaN-based "visible blind" or "solar blind" UV detectors to detect weak UV signals in the presence of strong ambient light in scientific research, industrial, and military applications. ${ }^{[21-29]}$ UV electroabsorption modulators have generally received much less attention. ${ }^{[30-36]}$

In this paper, we review progress in developing UV optoelectronic devices based on AlGaN alloys grown by plasmaassisted molecular beam epitaxy (PAMBE). These include LEDs, lasers, photodetectors, electroabsorption modulators, and distributed Bragg reflectors (DBRs).

\section{Growth, alloy ordering, and doping of AIGaN alloys}

The AlGaN alloys in the form of bulk films or multiple quantum wells (MQWs) as well as device structures based on such materials were deposited by PAMBE on the $c$-planes of sapphire and $6 \mathrm{H}-\mathrm{SiC}$ substrates. In this method, the molecular nitrogen is activated in a Veeco radiofrequency (RF) plasma source to produce active nitrogen species (atomic, ionic, and activated molecular nitrogen $\mathrm{N}_{2}{ }^{*}$ ) depending on the mode of operation. ${ }^{[37]}$ The $\mathrm{Ga}, \mathrm{Al}$, and In metals as well as $\mathrm{Si}$ and $\mathrm{Mg}$ dopants were supplied from standard effusion cells.

\section{Fundamental problems in AlGaN films}

There are a number of fundamental problems with AlGaN alloys independent of growth methods, which are responsible 
for the relatively low progress in developing efficient deep UV optoelectronic devices.

Due to the lack of native substrates such devices are generally grown heteroepitaxially primarily on the $c$-plane of sapphire and $6 \mathrm{H}-$ or $4 \mathrm{H}-\mathrm{SiC}$ substrates. So far, sapphire has been the substrate of choice because it is inexpensive and readily available in large sizes. Furthermore, sapphire is transparent in the entire UV spectral region. To mitigate problems related to large lattice mismatch (about 13\%) between AlGaN films and sapphire substrate a number of nucleation steps (conversion of the surface of sapphire from $\mathrm{Al}_{2} \mathrm{O}_{3}$ to $\mathrm{AlN}$ and the use of a low-temperature AlN buffer) have been developed to allow the annihilation of threading defects as the film grows thicker. ${ }^{[38-43]}$ However, in spite all of these the density of threading defects is still high $\left(\sim 10^{9} \mathrm{~cm}^{-2}\right)$.

There are multiple reasons for the high density of extended and point defects in heteroepitaxially grown AlGaN films. Principle among which is the high activity of nitrogen produced either by thermal decomposition of ammonia or by plasma decomposition of molecular nitrogen. ${ }^{[4]}$ During epitaxial growth this active nitrogen reacts instantly with arriving $\mathrm{Al}$ atoms on the substrate and limits their diffusivity. This leads to the nucleation of small $\mathrm{AlGaN}$ islands and films with microstructures consisting of small hexagonal columnar domains. Such materials are expected to have high dislocation density, since the plurality of the threading dislocations occur primarily at the boundaries of the hexagonal columnar domains due to their incomplete coalescence. ${ }^{[45]}$ Furthermore, coalescence of the small islands leads to tensile stress, which promotes the nucleation and propagation of cracks. ${ }^{[46]}$ This is even more important in silicon-doped high Al content AlGaN films, since silicon is an anti-surfactant and thus, it leads to microstructures with even smaller domains. ${ }^{[47]}$

Another source of the poor IQE of deep UV-LEDs is the incorporation of oxygen in AlGaN due to the high chemical affinity of aluminum for oxygen. ${ }^{[48-50]}$ While oxygen is a shallow donor in $\mathrm{GaN}$ and InGaN alloys, it is known to form DX-like centers in AlGaN alloys with high AlN mole fraction. ${ }^{[51]}$

Other potential problems are the poor doping efficiency of n- and particularly p-AlGaN with high AlN mole fraction, which is responsible for the poor carrier injection in the active region of the device. ${ }^{[52]}$ This is due to the high ionization energies of $\mathrm{Mg}$ acceptors and Si-donors, which are 630 and $280 \mathrm{meV}$ respectively for AlN. ${ }^{[20]}$

Yet, another fundamental problem is the difficulty in extracting the light from deep UV-LEDs due to emission selection rules originating from changes in the valence band structure of AlGaN alloys as the AlN mole fraction in the alloy increases. ${ }^{[53]}$ Specifically, the band structure of AlGaN alloys changes as a function of AlN mole fraction due to the difference in the crystal field splitting of $\mathrm{GaN}(+38 \mathrm{meV})$ and $\mathrm{AlN}(-219 \mathrm{meV})$. As a result of the reversal of the order of the valence bands recombination in $\mathrm{GaN}$ leads to light polarized with the $E \perp C$-axis (surface emission), while in AlN the light is polarized with the $E \| C$-axis (edge emission).
Various approaches are currently been pursued to address these problems. A number of groups are developing deep UV emitters (LEDs and lasers) by growing such devices pseudomorphically on AlN substrates. ${ }^{[9,14,17]}$ However, such substrates are currently available in small sizes and are prohibitively expensive. Monroy et al. have employed indium as a surfactant to promote two-dimensional (2D) growth during deposition of AlGaN alloys under slightly N-rich conditions. $^{[54]}$

\section{Growth mode of III-nitrides by PAMBE}

Growth of AlGaN alloys by PAMBE has the potential to address some of these problems. Existing literature assumes that the growth of nitride semiconductors by PAMBE is a physical vapor phase deposition method, involving the reaction on a heated substrate of Ga-vapor with molecular nitrogen, activated first by a RF or microwave plasma. However, contrary to the growth by MBE of traditional III-V compounds (arsenides, phosphides, etc.), which takes place under group-V-rich conditions, the growth of atomically smooth GaN takes place under group-III-rich conditions of growth. ${ }^{[55-57]}$ Growth of $\mathrm{GaN}$ under N-rich conditions leads to films with rough and faceted surface morphology. This is partly due to the low temperature of growth and partly because the nitrogen-activated species are highly reactive as discussed previously. Indeed theoretical studies indicate that the diffusion barrier for Ga adatoms on N-rich surface is $1.8 \mathrm{eV}$, while for Ga-saturated surface is only 0.4 $\mathrm{eV} .^{[58]}$

The growth of GaN by PAMBE under Ga-rich condition has been addressed recently in a number of theoretical papers. First-principles total energy calculations by Northrup et al. have shown that under Ga-rich conditions the excess $\mathrm{Ga}$ forms a laterally contracted Ga bilayer. ${ }^{[59]}$ Neugebauer et al. employed density-functional theory in combination with scanning tunneling microscopy to account for the smooth surface morphology of $\mathrm{GaN}$ films grown by PAMBE under Ga-rich conditions. ${ }^{[60]}$ These authors demonstrated that a thin metallic film on a semiconductor surface may open an efficient diffusion channel for lateral adatom transport. Thus, adatoms may prefer diffusion within this metallic layer rather than on the top of the surface.

Our group has proposed an alternative model to account for the role of the excess $\mathrm{Ga}$ in the surface of the growing GaN film. ${ }^{[50,61,62]}$ Specifically, we have proposed that under Ga-rich conditions the GaN growth by PAMBE is a liquid phase epitaxy (LPE) rather than physical vapor deposition. In other words growth takes place through the saturation with active nitrogen of the metallic $\mathrm{Ga}$ at the growing surface, followed by subsequent crystallization from the melt onto the $\mathrm{GaN}$ seed. It should be stressed that while in traditional LPE growth the driving force is the gradient temperature between the liquid and seed, in the proposed LPE mode of MBE growth of $\mathrm{GaN}$, the driving force is the concentration gradient of dissolved nitrogen in the liquid gallium due to the constant supply of active nitrogen from the plasma source. 
This model assumes that the solubility of active nitrogen species is high at the low temperatures employed during growth of nitride semiconductors by MBE. The thermodynamics of $\mathrm{GaN}$ growth from the melt has been studied extensively by the High Pressure Institute group in Poland. ${ }^{[63]}$ The enthalpy of dissociation of molecular nitrogen is very high $(9.77 \mathrm{eV})$. The molecular nitrogen adsorption on liquid Ga surface is a dissociative process and the energy barrier for this process is 5.3 $\mathrm{eV}$. Due to this high-energy barrier the solubility of molecular nitrogen $\left(\mathrm{N}_{2}\right)$ in $\mathrm{Ga}$ is only 1 at $\%$ at $1900 \mathrm{~K}$. Thus, the growth of $\mathrm{GaN}$ from the melt usually takes place at high temperatures in order to increase the solubility of molecular nitrogen in $\mathrm{Ga}$. Also, the growth takes place under high pressure of more than $15 \mathrm{kbar}$ in order to prevent the decomposition of $\mathrm{GaN}$ at these high temperatures. ${ }^{[63]}$

Contrary to the low solubility of molecular nitrogen, there is ample experimental evidence that active nitrogen produced by plasma decomposition of molecular nitrogen or by thermal decomposition of ammonia has a high solubility in gallium even at low temperatures. Specifically, there are reports of forming $\mathrm{GaN}$ by exposing liquid $\mathrm{Ga}$ to a nitrogen plasma at temperatures of about $700^{\circ} \mathrm{C}^{[64-66]}$ and forming epitaxial GaN quantum dots (QDs) by exposing Ga-droplets on the sapphire substrate to ammonia or RF nitrogen plasma at similar temperatures. ${ }^{[67,68]}$ From the various active nitrogen species the metastable molecular nitrogen $\left(\mathrm{N}_{2} *\right)$ is expected to have the higher solubility in Ga since its internal energy is 9.5 $\mathrm{eV}^{[44]}$ and thus, its energy barrier to adsorption to Ga should be very small. On the other hand, the solubility of atomic nitrogen in Ga may be limited since Ga can catalyze the recombination of atomic nitrogen to form molecular nitrogen. Thus, the $\mathrm{N}_{2} *$ radicals are the species, which contribute mostly to the $\mathrm{GaN}$ growth at these low temperatures.

While the growth of GaN under Ga-rich conditions has been extensively investigated both theoretically and experimentally, the growth of $\mathrm{AlGaN}$ under Ga-rich conditions raises the issue of how the excess $\mathrm{Ga}$ in the surface of the AlGaN film affects the film stoichiometry. The kinetics of growth of AlGaN alloys by PAMBE has previously reported by Iliopoulos and Moustakas. ${ }^{[69]}$ These authors reported that the stoichiometry of the films depends only on the ratio of the [Al] to active $[\mathrm{N}]$ fluxes $([\mathrm{Al}] /[\mathrm{N}])$ and not by the ratio of $[\mathrm{Al}] /[\mathrm{Ga}]$ fluxes as it is the case in AlGaAs. ${ }^{\left[{ }^{[0]}\right.}$ This is due to the much stronger $\mathrm{Al}-\mathrm{N}$ bond versus the $\mathrm{Ga}-\mathrm{N}$ bond. Thus, any excess gallium during growth accumulates on the surface of the growing AlGaN film in the form of liquid gallium, but it does not affect the film stoichiometry. In the presence of excess $\mathrm{Ga}$ in the surface of the growing $\mathrm{AlGaN}$ films the growth proceeds via LPE as discussed previously. In other words, the arriving active nitrogen species, $\mathrm{Al}$ atoms, as well as intentional or unintentional impurities dissolve first in the liquid $\mathrm{Ga}$ and incorporate into the AlGaN film from the liquid phase.

The growth of AlGaN by PAMBE under Ga-rich conditions has a number of beneficial effects in the optoelectronic quality of these films. This growth mode is likely to lead to lateral compositional inhomogeneities due to statistical fluctuations of the thickness of the liquid Ga on the surface of the growing AlGaN film. These compositional inhomogeneities lead to band-structure potential fluctuations, which are sufficiently deep that lead to exciton localization even at room temperature. This prevents the carriers from diffusing to point or extended defects and to recombine non-radiatively. ${ }^{[71]}$ Another source of band-structure potential fluctuations is the partial alloy ordering, which is discussed below.

Also, in the proposed LPE growth mode the incorporation of impurities in the AlGaN film requires that their solubility in $\mathrm{Ga}$ at the growth temperature to be relatively high. We have reported previously that the concentration of impurities such as $\mathrm{O}, \mathrm{H}$, and $\mathrm{C}$ is in the $10^{19} \mathrm{~cm}^{-3}$ range when the GaN films are grown under nitrogen-rich conditions, while they are two to three orders of magnitude less when the films are grown under Ga-rich conditions. ${ }^{[50,62]}$ The reduction in oxygen incorporation can be accounted for by the formation of volatile gallium oxides, which would then desorb. Similarly hydrogen may form volatile gallium hydrides. Regarding the reduction in carbon impurities one has to assume that the solubility of carbon in Ga must be very low, since gallium carbides compounds are not known to exist. The incorporation of dopant impurities such as Si and $\mathrm{Mg}$ is discussed below.

It is important to stress that in general the LPE growth process has been successfully treated as a thermodynamic process. ${ }^{[72]} \mathrm{A}$ thermodynamic analysis of $\mathrm{Al}$ incorporation in $\mathrm{GaN}$ toward the formation of $\mathrm{AlGaN}$ during MBE growth of nitride semiconductors under metal-rich conditions was presented by Hoke et al. ${ }^{[73]}$ As discussed previously the active nitrogen dissolves in the liquid $\mathrm{Ga}$ in the surface of the film and incorporates in the GaN seed from the liquid phase. If simultaneously $\mathrm{Al}$ atoms arrive on the substrate, then the incorporation of $\mathrm{Al}$ is favored, if the Gibbs free energy at the growth temperature $\left(\Delta G^{\mathrm{T}}\right)$ of the reaction in Eq. (1) is negative.

$$
\mathrm{Al}+\mathrm{GaN} \rightarrow \mathrm{AlN}+\mathrm{Ga}, \Delta G^{\mathrm{T}}<0 .
$$

The Gibbs free energies of formation at $1000 \mathrm{~K}$ for a number of relevant compounds and elements are given in Table I of Ref. 73. Using these values we find $\Delta G^{\mathrm{T}}=-209.6 \mathrm{~kJ} / \mathrm{mole}$ and thus, this reaction is strongly thermodynamically favorable. Therefore, Al will preferentially incorporate into the growing nitride film despite the excess $\mathrm{Ga}$ in the surface. As discussed previously, this is in agreement with the findings in Ref. 69, that the composition of $\mathrm{Al}_{x} \mathrm{Ga}_{1-x} \mathrm{~N}$ alloys is determined by the ratio of the incoming $\mathrm{Al}$ flux to the reactive nitrogen flux $[\mathrm{Al}] /[\mathrm{N}]$. In the limit of $[\mathrm{Al}]=[\mathrm{N}]$ pure AlN film will grow despite the excess $\mathrm{Ga}$ in the surface of the growing film.

\section{Alloy ordering in AIGaN films}

The phenomenon of long-range atomic ordering has been observed in a wide range of III-V semiconductor alloy systems. ${ }^{[74]}$ The most studied case of ordering is probably the CuPt-type in the alloy system GaInP. ${ }^{[75]}$ In an ideally ordered 
case, instead of a random $\mathrm{Ga}_{0.5} \mathrm{In}_{0.5} \mathrm{P}$ alloy, a $(\mathrm{GaP})_{1}(\mathrm{InP})_{1}$ superlattice (SL) is formed spontaneously in the [111]-direction during growth on the (001) plane. Long-range atomic ordering in cubic III-V alloys is found to be driven by surface phenomena and not by bulk thermodynamics. ${ }^{[76]}$ In the case of cubic III-V alloys, theoretical calculation and experimental evidence attribute the phenomenon to specific surface reconstructions caused by subsurface strain. ${ }^{[77]}$ Such reconstructions arrange adatoms on the surface $\{001\}$ planes in such a way that a SL is created along the $\{111\}$ planes, when these adatoms are subsequently buried. The formation of the ordered phase affects both the alloy optical ${ }^{[75]}$ and electronic properties. ${ }^{[78]}$

Long-range atomic ordering in $\mathrm{AlGaN}$ films grown by PAMBE on the $c$-plane of sapphire and $6 \mathrm{H}$-SiC substrates were first reported by Korakakis et al. ${ }^{[79]} \mathrm{Al}_{x} \mathrm{Ga}_{1-x} \mathrm{~N}$ alloys crystallize in the wurtzitic, hexagonal close-packed structure. The geometrical structure factor for a Bragg reflection $(h k l)$ in such crystals is given by the expression:

$$
F_{h k l}=f_{\mathrm{A}}+f_{\mathrm{B}} \exp [2 \pi i[(h+2 k) / 3+l / 2]],
$$

where $f_{\mathrm{A}}$ and $f_{\mathrm{B}}$ are the average scattering factors of the $\mathrm{Al}, \mathrm{Ga}$ atoms occupying the (000) and $(1 / 3,2 / 3,1 / 2)$ sublattice sites of the hexagonal cell, respectively. In Eq. (2), we have omitted the scattering from the nitrogen atoms since their presence does not affect the discussion related to cation ordering. When the two sites are occupied by the same atomic species or by a random mixture of the two species, Bragg reflections with $1=$ odd and $h+2 k=3 n$ are forbidden according to Eq. (2). Thus, in pure $\mathrm{GaN}$, pure $\mathrm{AlN}$, and random $\mathrm{Al}_{x} \mathrm{Ga}_{1-x} \mathrm{~N}$ alloys, the (0001), (0003), etc. diffractions are forbidden. However, if one of the sublattice sites is preferentially occupied by $\mathrm{Al}$ or $\mathrm{Ga}$ in an $\mathrm{Al}_{x} \mathrm{Ga}_{1-x} \mathrm{~N}$ alloy, the two terms no longer cancel and SL peaks result.

An example of such an x-ray diffraction (XRD) was reported by Iliopoulos et al. ${ }^{[80]}$ and is shown in Figure 1 for an $\mathrm{Al}_{0.65} \mathrm{Ga}_{0.35} \mathrm{~N}$ grown by PAMBE on the $c$-plane of sapphire. Apart from the allowed (0002) $\mathrm{AlGaN}$ and (0006) sapphire peaks, the reflections at the (0001) and the (0003) AlGaN reciprocal space points are present. It is important to note that such experimental data cannot be attributed to stacking faults in the films, since the geometrical structure factor for reflections with indices $h=k=0$ are independent of the basal plane coordinates of the atoms in the unit cell and therefore cannot probe the stacking sequence of planes. Instead the (0001) and (0003) peaks are a direct indication of the presence of $1 \times 1$ ordering in the crystal. Also to rule out atomic ordering due to vacancies or strain we have confirmed the long-range atomic ordering in these films by polarization-dependent EXAFS. ${ }^{[81]}$

In contrast to the case with the cubic arsenides and phosphides, the ordering reported in the wurtzite nitride alloys occurs along the [0001] growth direction. Therefore the mechanisms that induce ordering in the arsenides and phosphides would appear not to be applicable in the nitrides. Theoretical and experimental investigations of the formation mechanism(s)

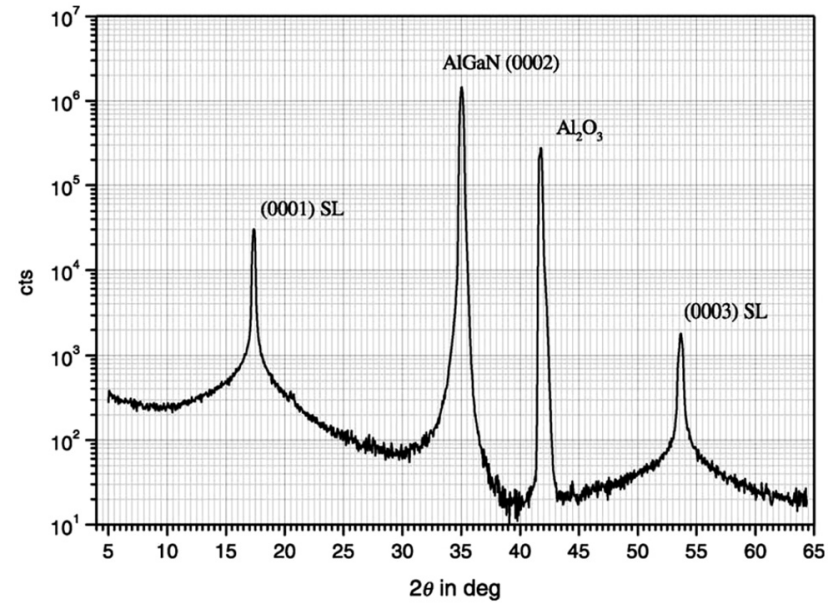

Figure 1. On-axis XRD scan of an $\mathrm{Al}_{0.55} \mathrm{Ga}_{0.45} \mathrm{~N}$ film grown by $\mathrm{RF}$ PAMBE. ${ }^{[80]}$

for ordering in the nitrides have been limited. However, a model to explain simple $1 \times 1$ ordering with alternating Gaand Al-rich layers, has been proposed by Northrup et al. ${ }^{[82]}$

In addition to $1 \times 1$ atomic ordering, Iliopoulos et al. have reported complex ordering in the nitrides along the [0001] growth direction, with periodicities of several wurtzite unit cells. ${ }^{[80,83,84]}$ This differs qualitatively from the $1 \times 1$ ordering discussed previously, which does not alter the fundamental structural repeat distance along the $c$-direction. An example of such complex ordering structure is shown in Figure 2. Figure 2(a) is the on-axis $\theta-2 \theta \mathrm{XRD}$ spectra for three $c$-plane $\mathrm{Al}_{x} \mathrm{Ga}_{1-x} \mathrm{~N}$ films grown by PAMBE on the $c$-plane sapphire for differing group-III beam equivalent pressure (BEP) values. Here the BEP is the sum of the Al and Ga fluxes, while their ratio was held fixed $\left(\mathrm{BEP}_{\mathrm{Al}} / \mathrm{BEP}_{\mathrm{Ga}}=0.53\right)$. In particular, samples $\mathrm{A}, \mathrm{B}$, and $\mathrm{C}$ were grown with III/V flux ratios of 1.0, 0.9, and 0.6 , respectively. As measured by RBS, the final compositions of the films were $x=0.89$ for sample A, $x=0.74$ for sample $\mathrm{B}$, and $x=0.55$ for sample $\mathrm{C}^{[83]}$ Otherwise the three samples were grown with identical nucleation and growth conditions.

Sample C, which was grown under N-rich conditions of growth, shows the (0001) diffraction peak characteristic of the simple $1 \times 1$ ordered structure discussed above. However, the films grown under group-III-rich conditions show a number of additional SL peaks indicating the presence of a SL structure with different periodicity.

Film A of Figure 2(a) was also studied by transmission electron microscope (TEM) selected area diffraction (SAD). The SAD pattern in Figure 2(b) shows the same SL peaks observed by the XRD in Figure 2(a). In taking the data described in Figure 2(b) the zone axis $\langle 11-20\rangle$ was tilted toward the $\langle 10$ $10\rangle$ direction to avoid any double diffraction. ${ }^{[84]}$

As discussed by Iliopoulos et al., ${ }^{[83]}$ the XRD and TEM data of sample A can be accounted for by the presence of two 

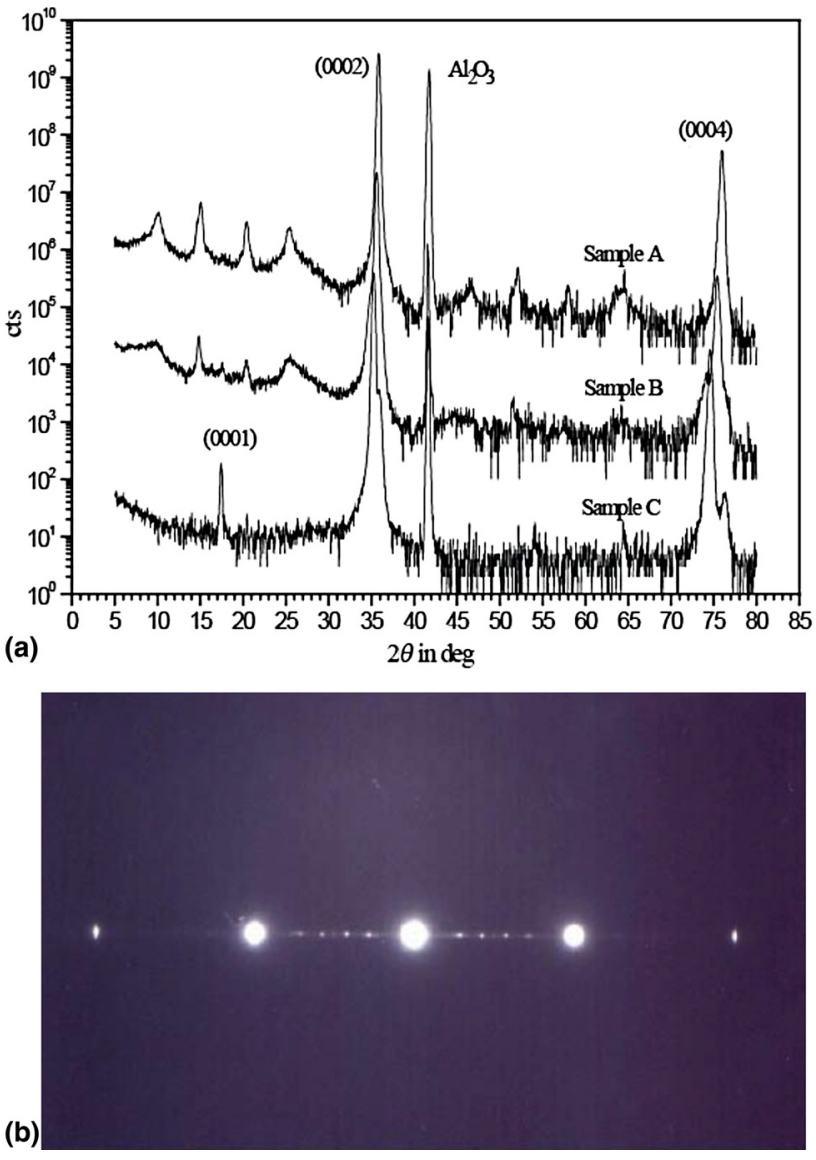

Figure 2. (a) On-axis XRD for three AIGaN films grown with different ratios of III/V fluxes; (b) SAD pattern of the AIGaN film A. ${ }^{[83]}$

spontaneously formed SL structures in the film: a dominant one having a period of 14-ML and a secondary one having a period of 12-ML.

However, Wang et al. ${ }^{[85]}$ reported that $\mathrm{Al}_{0.72} \mathrm{Ga}_{0.28} \mathrm{~N}$ films grown in environments with group-III/N ratios $>1$ exhibit ordered SL structures that are incommensurate with the wurtzite crystal lattice. The increasing complexity of the ordering with increasing Ga-rich growth environment suggests that the ordering is related to the presence of a $\mathrm{Ga}$ overlayer.

Other groups have also reported the existence of complex ordered structures, ${ }^{[86,87]}$ but with repeat distances of 3,4 , and 6 monolayers, sometimes coexisting in a single sample. In these references, the films were grown by the metalorganic chemical vapor deposition (MOCVD) method, suggesting that this type of complex ordering in nitride alloys is universal and depends sensitively on growth conditions.

It should be stressed that the observed atomic ordering in both $\mathrm{AlGaN}$ and InGaN alloys is only partial. ${ }^{[79]}$ In other words, some domains are atomically ordered and some are random. ${ }^{[88]}$ Also, since the energy gap of the alloy was found to depend on the degree of ordering, ${ }^{[89]}$ partial atomic ordering introduces band-structure potential fluctuations, which may have an effect on the performance of the optoelectronic devices as discussed below.

\section{Doping}

Silicon and magnesium are the primary n- and p-type dopants in GaN and its alloys with AlN and InN.

\section{N-type doping}

There are multiple reports that the conductivity in these n-type doped alloys decreases drastically with AlN mole fraction. For example, in unintentionally n-type doped AlGaN alloys grown by MOCVD, Lee et al. ${ }^{[90]}$ reported a rapid decrease in conductivity for AlN mole fraction higher than 40\%. McCluskey et al. ${ }^{[91]}$ attributed the unintentional n-type conductivity to oxygen impurities. Bremser et al., ${ }^{[92]}$ using also the MOCVD method, have doped AlGaN alloys with silicon up to AlN mole fraction of $42 \%$. However, additional silicon resulted in highly resistive films. Taniyasu et al. ${ }^{[93]}$ reported that AlGaN films containing more than $3 \times 10^{19} \mathrm{~cm}^{-3}$ of silicon become highly resistive due to self-compensation of silicon donors. Skierbiszewski et al. ${ }^{[94]}$ studied AlGaN films with AlN mole fraction between $50 \%$ and $60 \%$ under high pressure. These authors concluded that the Si dopant forms two donor states: one of them is shallow and has an effective mass character; the other is strongly coupled to the crystal lattice and thus, forms a deeper state. They also argued that this metastable localized state is the source for persistent photoconductivity in high $\mathrm{Al}$ concentration AlGaN alloys. Stampfl et al. ${ }^{[95]}$ concluded, based on density-functional-pseudopotential calculations, that the experimentally observed decrease in n-type conductivity for AlN mole fraction more than $40 \%$ has two potential origins: (a) for auto-doped films (doped unintentionally with oxygen) the reduction in conductivity is due to a DX transition that converts the shallow donor into deep level; (b) in the case of silicon the reduction of conductivity is due to cation vacancies. The Cornell and the Ohio State groups ${ }^{[96,97]}$ have reported significant progress in doping AlGaN alloys with silicon by PAMBE. Specifically, they were able to dope AlGaN alloys with $80 \%$ AlN mole fraction to a level of $8.5 \times 10^{19} \mathrm{~cm}^{-3}$ electron concentration.

The incorporation of $\mathrm{Si}$ in nitride semiconductors should follow the thermodynamic analysis, which was discussed previously. ${ }^{[73]}$ According to this model the incorporation of Si in GaN and AlN can be determined from Eqs. (3) and (4), using the Gibbs free energies from Ref. 73.

$$
\begin{aligned}
\mathrm{Si}+4 / 3 \mathrm{GaN} & \rightarrow 1 / 3 \mathrm{Si}_{3} \mathrm{~N}_{4}+4 / 3 \mathrm{Ga}, \\
\Delta G^{1000 \mathrm{~K}} & =-134.7 \mathrm{~kJ} / \text { mole } \\
\mathrm{Si}+4 / 3 \mathrm{AlN} & \rightarrow 1 / 3 \mathrm{Si}_{3} \mathrm{~N}_{4}+4 / 3 \mathrm{Al}, \\
\Delta G^{1000 \mathrm{~K}} & =+144.7 \mathrm{~kJ} / \text { mole. }
\end{aligned}
$$

As pointed out in Ref. 73 these equations do not imply that a $\mathrm{Si}_{3} \mathrm{~N}_{4}$ molecule exists on the surface of the growing film but 
that the strength of the $\mathrm{Si}-\mathrm{N}$ bonds in the film is reflected in the free energy of formation of this molecule. Based on these thermodynamic arguments, under Ga-rich growth conditions $\mathrm{Si}$ should incorporate and dope GaN n-type. On the other hand, under Al-rich conditions $\mathrm{Si}$ cannot incorporate in AlN. Doping of AlN with Si was reported only during growth of AlN under N-rich conditions. ${ }^{[98]}$

Our group has reported the degenerate n-type doping with $\mathrm{Si}$ by PAMBE of AlGaN films up to $80 \%$ AlN mole fraction. ${ }^{\text {[9] }}$ Figure 3 shows the dependence of carrier concentration and electron mobility on AIN mole fraction. The Si cell temperature during growth of these films was kept at constant temperature.

\section{P-type doping}

The doping of GaN and its alloys p-type with $\mathrm{Mg}$ cannot be done easily under $\mathrm{N}$-rich conditions because at the growth temperatures of $\mathrm{GaN}$ of about $700{ }^{\circ} \mathrm{C}$ the vapor pressure of $\mathrm{Mg}$ is more than 10 Torr and thus, the sticking coefficient of $\mathrm{Mg}$ will
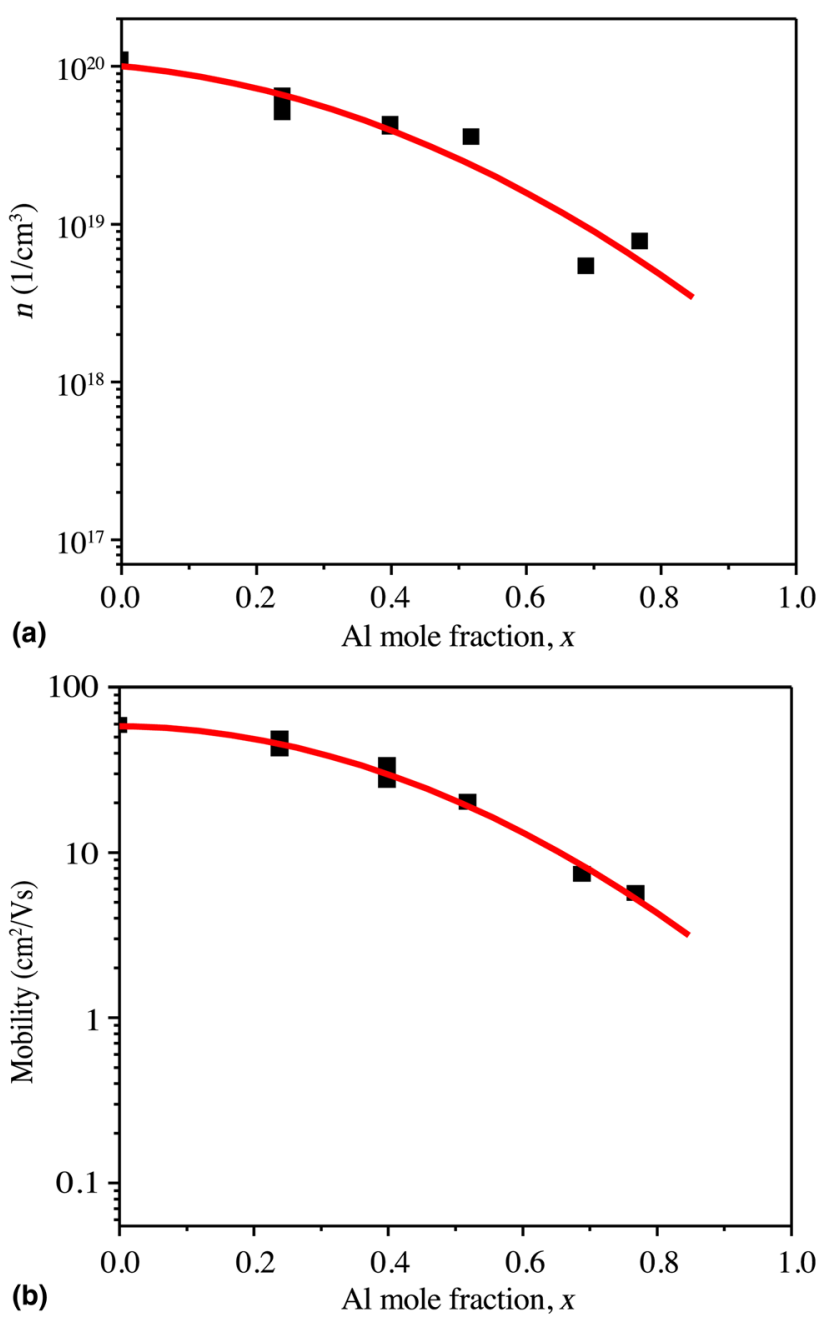

Figure 3. The dependence of (a) carrier concentration and (b) electron mobility on AIN mole fraction in Si-doped AIGaN films grown by PAMBE. ${ }^{[99]}$ be extremely small. ${ }^{[100]}$ According to the thermodynamic model, based on Eq. (5), Mg should readily incorporate into $\mathrm{GaN}$ under Ga-rich conditions of growth.

$$
\begin{aligned}
\mathrm{Mg}+2 / 3 \mathrm{GaN} & \rightarrow 1 / 3 \mathrm{Mg}_{3} \mathrm{~N}_{2}+2 / 3 \mathrm{Ga}, \\
\Delta G^{1000 \mathrm{~K}} & =-79.3 \mathrm{~kJ} / \text { mole }
\end{aligned}
$$

Bhattacharyya et al. demonstrated efficient p-doping of GaN with $\mathrm{Mg}$ when growth takes place under Ga-rich conditions. ${ }^{[100]}$ Figure 4 shows the resistivity versus $\mathrm{Mg}$ cell temperature for two series of $\mathrm{p}-\mathrm{GaN}$ films grown at $770{ }^{\circ} \mathrm{C} .{ }^{[100]}$

As indicated in Figure 4, the one series of $\mathrm{p}-\mathrm{GaN}$ films was grown under Ga-rich conditions, while the second one was grown under stoichiometric conditions. The poorer incorporation efficiency of $\mathrm{Mg}$ in $\mathrm{GaN}$ grown under stoichiometric conditions was attributed to the low sticking coefficient of $\mathrm{Mg}$ as discussed previously. On the other hand, during growth under Ga-rich conditions the $\mathrm{Mg}$ dissolves in the liquid gallium on the surface of $\mathrm{GaN}$ and incorporates from the liquid phase. Based on this argument $\mathrm{GaN}$ can be doped more efficiently p-type with $\mathrm{Mg}$ under Ga-rich conditions at higher temperatures, since the solubility of $\mathrm{Mg}$ in $\mathrm{Ga}$ is higher.

A number of $\mathrm{p}-\mathrm{GaN}$ films were grown under Ga-rich conditions in our laboratory and their transport coefficients were evaluated by Hall Effect measurements. As shown in Figure 5, the hole mobility versus hole concentration follows the expected monotonic relation.

As discussed previously the p-doping with $\mathrm{Mg}$ of $\mathrm{AlGaN}$ films with high AlN mole fraction is limited by the high ionization energy of $\mathrm{Mg}$ acceptors. An alternative approach to improve the doping efficiency of $\mathrm{AlGaN}$ is to dope the material in the form of an $\mathrm{Al}_{x} \mathrm{Ga}_{1-x} \mathrm{~N} / \mathrm{Al}_{y} \mathrm{Ga}_{1-y} \mathrm{~N} \mathrm{SL} .{ }^{[101]}$ The concept of

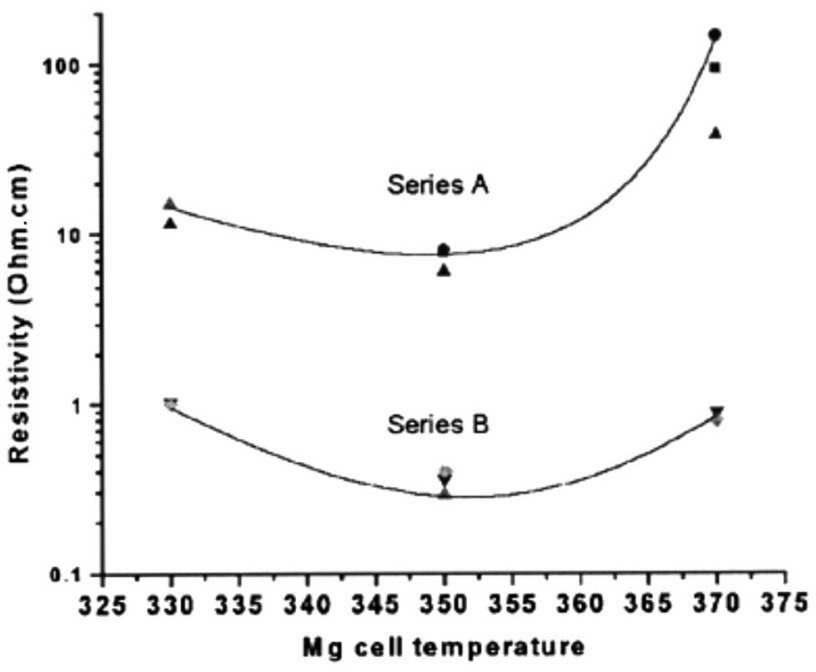

Figure 4. Resistivity versus Mg cell temperature for two families of $p-G a N$ films grown as discussed in the text. ${ }^{[100]}$ 


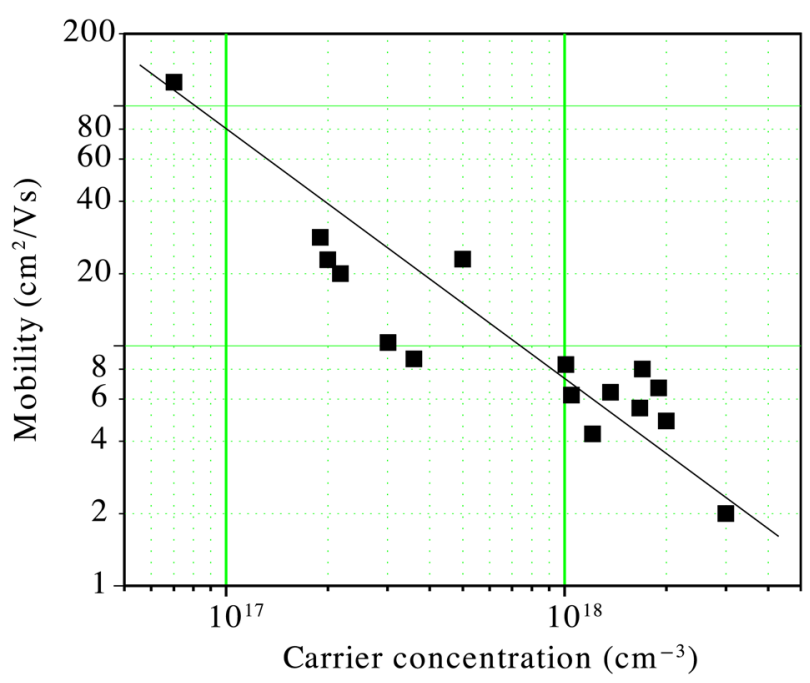

Figure 5. Mobility versus carrier concentration for a number of $p$-GaN films grown under Ga-rich conditions.

SL doping was first proposed in 1990 by Suemune to improve the acceptor doping efficiency in II-VI compounds. ${ }^{[102]}$ These calculations predicted a hole concentration for the SL a factor of five higher than that of the bulk ZnSe films. In 1999, a number of groups have applied the concept to doping with $\mathrm{Mg}$ of AlGaN/GaN SLs and reported an order of magnitude higher hole concentration than the corresponding AlGaN films with the same average composition. ${ }^{[103-105]}$ Figure 6 shows the resistivity versus AlN mole fraction for bulk AlGaN films and $\mathrm{Al}_{x} \mathrm{Ga}_{1-x} \mathrm{~N} / \mathrm{Al}_{y} \mathrm{Ga}_{1-y} \mathrm{~N}$ SLs grown by our group using PAMBE. ${ }^{[101]}$ It is obvious from these data that SL doping is far more efficient.

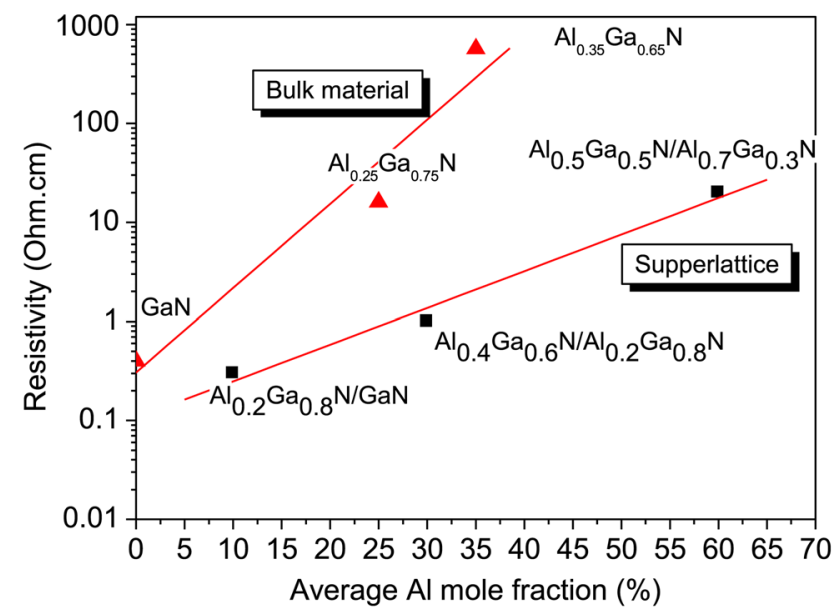

Figure 6. Resistivity versus average AIN mole fraction for Mg-doped bulk AlGaN films and $\mathrm{Al}_{x} \mathrm{Ga}_{1-x} \mathrm{~N} / \mathrm{Al}_{y} \mathrm{Ga}_{1-y} \mathrm{~N}$ SLs. ${ }^{[101]}$

\section{Development of AlGaN QWs with high IQE}

As discussed previously there are a number of potential advantages of growing $\mathrm{AlGaN}$ alloys by PAMBE under excess Ga. In this section, we are investigating of how this growth mode affects the IQE of AlGaN MQWs, which are the active regions of UV-LEDs and lasers. Bhattacharyya et al. investigated the effect of the excess liquid-Ga on the IQE of AlGaN films by growing a series of identical in thickness and composition $\mathrm{Al}_{0.70} \mathrm{Ga}_{0.30} \mathrm{~N} / \mathrm{AlN}$ MQWs on the $c$-plane of sapphire substrates and studying their photoluminescence (PL) efficiency as a function of temperature. ${ }^{[71]}$ Specifically, during the growth of the wells the flux of Ga was varied from that corresponding to stoichiometric conditions (III/V $\sim 1$ ) to (III/V $\gg 1$ ). The thickness of the barriers and wells were 6 and $1.5 \mathrm{~nm}$, respectively.

The normalized luminescence spectra of these samples are presented in Figure 7. These data show that all MQW samples exhibit only a sharp near band-edge emission. It is important to stress that although the thicknesses and compositions of the wells and barriers were the same for all samples, the emission wavelengths varies from 225 to $250 \mathrm{~nm}$ as the Ga-flux increased from III/V close to one to much greater than one. This red shift of the PL spectra for these identical MQWs indicates that the excess Ga during the growth of the wells introduces band-structure potential fluctuations whose depth increases with the amount of excess Ga. Consistent with this interpretation is also the increase of the spectral width from 11 to $16 \mathrm{~nm}$. These potential fluctuations are much deeper than the statistical ones due to alloy disorder and thus, they can cause carrier localization and efficient radiative recombination even at room temperature. The origin of these bandstructure potential fluctuations are either compositional

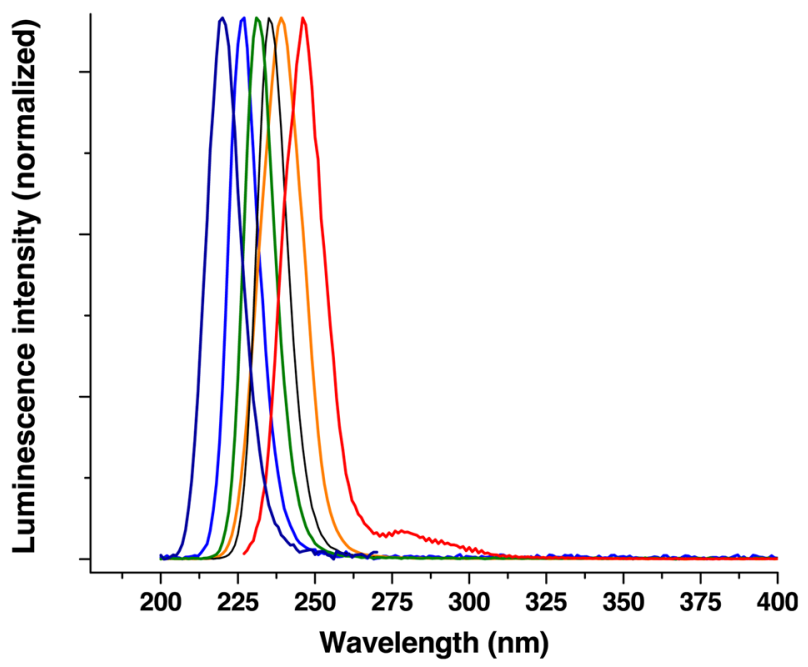

Figure 7. Room-temperature luminescence from $\mathrm{Al}_{0.7} \mathrm{Ga}_{0.3} \mathrm{~N} / \mathrm{AIN} \mathrm{MQWs}$, with identical well and barrier widths as described in the text. ${ }^{[71]}$ 
inhomogeneities due to statistical fluctuations of the thickness of Ga covering the surface of the growing AlGaN film or are due to partial alloy ordering as discussed previously.

The IQE of these MQWs was determined by measuring the PL spectra as a function of temperature. We define the IQE at room temperature as the ratio of the integrated PL intensity at room temperature divided by that at $10 \mathrm{~K} .^{[106]}$ This definition assumes that the recombination at $10 \mathrm{~K}$ is radiative, which is a reasonable assumption. The IQEs for the samples discussed in Figure 7 are presented in Figure 8. Thus, the IQE for these MQWs varies from $5 \%$ for emission at $225 \mathrm{~nm}$, to $50 \%$ for emission at $250 \mathrm{~nm}$. This increase in the IQE for identical $\mathrm{Al}_{0.7} \mathrm{Ga}_{0.3} \mathrm{~N} / \mathrm{AlN} \mathrm{MQWs}$ is attributed to the localization of the excitons due to band-structure potential fluctuations introduced during the growth of the wells under Ga-rich conditions.

Our group also investigated the growth and properties of AlGaN MQWs emitting in the deep UV on the $c$-plane of $6 \mathrm{H}-$ and $4 \mathrm{H}-\mathrm{SiC}$ substrates. ${ }^{[107]}$ Growth of AlGaN alloys and QWs on SiC for emitters has several advantages. The lattice mismatch between $\mathrm{SiC}$ and $\mathrm{AlN}$ is only $\sim 1 \%$. Other advantages include the high thermal conductivity of $\mathrm{SiC}$ compared with that of sapphire, as well as the ability to form facets by cleaving. Simultaneously, the growth of AlGaN on $\mathrm{SiC}$ substrates has a number of challenges, principal among which are the accidental nitridation of the $\mathrm{SiC}$ substrate prior to the epitaxial growth and the formation of stacking mismatch boundaries at the step edges due to the polytype difference between $2 \mathrm{H}-\mathrm{AlGaN}$ and $6 \mathrm{H}-$ or $4 \mathrm{H}-\mathrm{SiC} .{ }^{[108]}$ Zhang et al. reported that such AlGaN/ AIN MQWs emitting at $245 \mathrm{~nm}$ have an IQE of $68 \%$. ${ }^{[107]}$

These values of IQE for AlGaN MQWs emitting at $250 \mathrm{~nm}$ are comparable with those of InGaN MQWs. ${ }^{[106]}$ In the case of InGaN QWs, the high IQE is generally attributed to band-structure potential fluctuations due to compositional

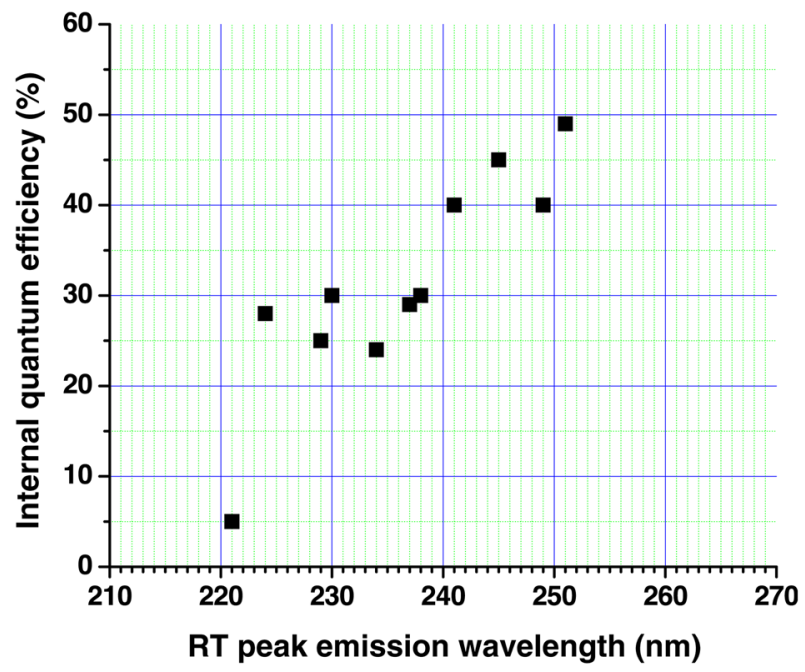

Figure 8. Room-temperature IQE of the $\mathrm{Al}_{0.70} \mathrm{Ga}_{0.30} \mathrm{~N} / \mathrm{AIN}$ MQWs described in Figure $7 .^{[71]}$ inhomogeneities in these alloys. The origin of these compositional inhomogeneities is phase separation by spinodal decomposition due to the $11 \%$ difference in the ionic radius of In and $\mathrm{Ga}$ atoms in tetrahedral sites. On the other hand, such phase separation is not expected in $\mathrm{AlGaN}$ alloys since $\mathrm{Al}$ and $\mathrm{Ga}$ atoms have identical ionic radius. Thus, we believe that the band-structure potential fluctuations in these alloys are the result of the growth mode under excess Ga as discussed earlier.

\section{UV-LEDs}

The majority of the UV-LEDs reported in the literature were produced by the MOCVD method. ${ }^{[5-10]}$ The MBE method has a number of advantages in developing such devices. As discussed earlier this method has the ability to introduce bandstructure potential fluctuations in the active region of the device, which promote efficient radiative recombination. Furthermore, it can prevent the incorporation of certain undesirable impurities such as oxygen, carbon, and hydrogen, and also it can facilitate the incorporation of dopant impurities such as $\mathrm{Mg}$ and $\mathrm{Si}$. In this section, we report the progress made in developing such devices by PAMBE. Our group initially developed efficient UV-LEDs emitting in the $340-350 \mathrm{~nm}$ as well as methods of growing such devices on textured GaN templates to improve both the IQE and EE. ${ }^{[109,110]}$

More recently, in a series of papers Liao et al. reported for the first time the development of $\mathrm{mW}$ power deep UV-LEDs grown by MBE on sapphire substrates. ${ }^{[111-114]}$ The epilayer structure of the investigated deep UV-LED devices, is schematically shown in Figure 9.

The AlN template was approximately $2 \mu \mathrm{m}$ thick. A 10 -period AlGaN/AlN strain management SL was grown on to the AlN template before the growth of the Si-doped

\section{$\mathrm{Ni}-\mathrm{Au}$ contact}

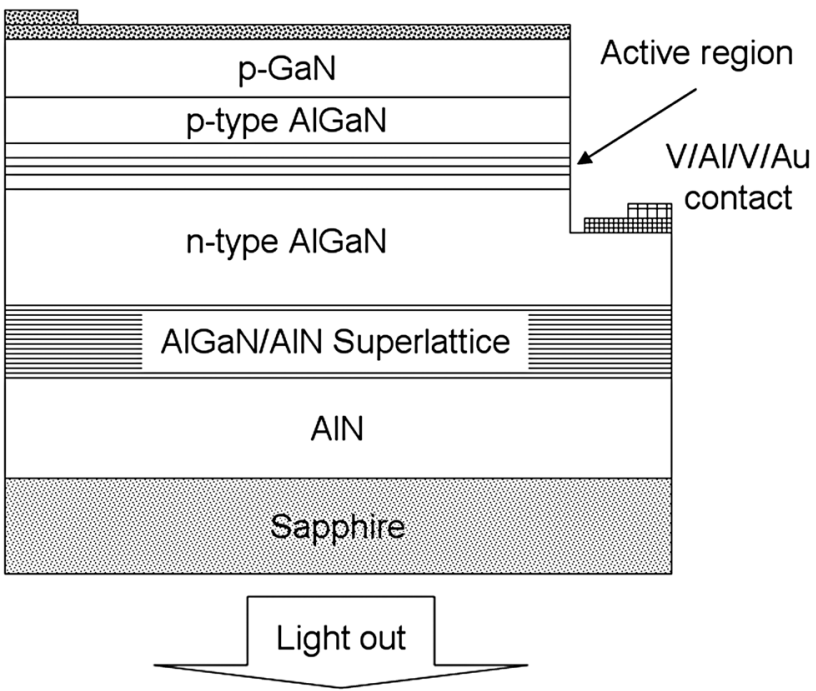

Figure 9. Schematic representation of the epitaxial design of the deep UV-LEDs investigated. ${ }^{[111]}$ 
n-AlGaN cladding layer. Such strain management SL has been proved to be effective in accommodating the elastic strain induced by $\mathrm{Si}$ incorporation into the $\mathrm{AlGaN}$ lattice and to prevent cracking. ${ }^{[115,116]}$ The electron concentration of the Si-doped $\mathrm{n}-\mathrm{AlGaN}$ was of the order of mid- $10^{18} \mathrm{~cm}^{-3}$. The active region consisted of a single asymmetric AlGaN/AlGaN quantum well (QW) and a 10-nm AlGaN electron-blocking layer (EBL) heavily doped with $\mathrm{Mg}$. The thickness of the barrier close to n-AlGaN layer was $10 \mathrm{~nm}$, while the other barrier next to EBL was $3 \mathrm{~nm}$. Such an asymmetric design was chosen in order to balance the electron and hole injections into the QW due to differences in diffusion lengths of holes and electrons. ${ }^{[117]}$ The composition and thickness of the EBL were optimized in order to best block electron overflow into the p-type region. The AlGaN QW was grown under Ga-rich conditions to allow for the formation of band-structure potential fluctuations. The final structure is capped by a p-AlGaN layer and $\mathrm{p}-\mathrm{GaN}$ contact layer. The Al composition of the various layers was varied depending of the intended emission wavelength of the device. Ohmic contacts to $\mathrm{n}-\mathrm{AlGaN}$ were made using vanadium-based alloys whose contact resistivity was reported previously to be $10^{-6} \Omega / \mathrm{cm}^{2}$ for AlN mole fraction up to $70 \%$. ${ }^{[118-121]}$

Liao et al. demonstrated the dependence between IQE and band-structure potential fluctuations by studying the optical absorption and the PL spectra of two identical $\mathrm{Al}_{0.5} \mathrm{Ga}_{0.5} \mathrm{~N} /$ $\mathrm{Al}_{0.65} \mathrm{Ga}_{0.35} \mathrm{~N}$ MQWs, grown under different amount of excess Ga. ${ }^{[13]}$ The one MQW was grown under Ga-rich conditions (III/V > 1, MQW-1) and the second was grown under stoichiometric conditions (III/V 1, MQW-2). The results are shown in Figure 10. From these data we have estimated the IQE of MQW-1 to be $32 \%$ and that of the MQW-2 to be $15 \%$. The inset of Figure 10 shows the derivative of the optical

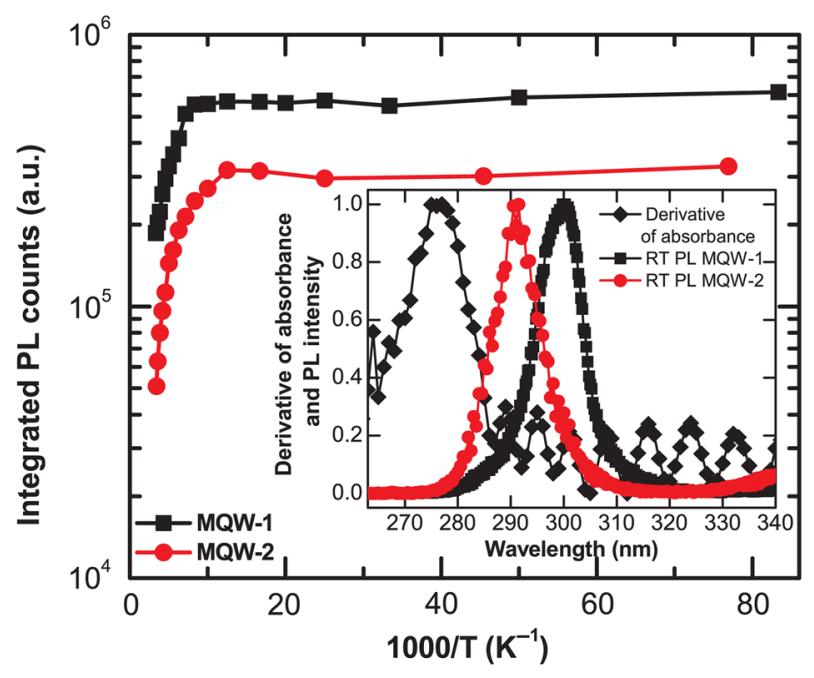

Figure 10. Plot of integrated PL intensity versus inverse temperature of MQW-1 and MQW-2; inset shows derivative of absorbance of samples MQW-1 and -2 , and their RT emission spectra. ${ }^{[113]}$ absorbance obtained from transmission measurements from these MQWs and their RT PL spectra. The peak of the derivative of the absorption constant at $275 \mathrm{~nm}$ is a measure of the optical gap of the $\mathrm{Al}_{0.5} \mathrm{Ga}_{0.5} \mathrm{~N} .{ }^{[41]}$ As seen from this data the peak of the PL spectra at $300 \mathrm{~nm}$ is red shifted from the optical band edge by $25 \mathrm{~nm}(376 \mathrm{meV})$. Such large Stokes shift is another evidence of band-tail states due to potential fluctuations. In contrast, the Stokes shift of MQW-2 is only $15 \mathrm{~nm}$ (233 $\mathrm{meV}$ ). The correlation between IQE and Stokes shift of the two samples grown with and without excess Ga clearly establishes that excess $\mathrm{Ga}$ during $\mathrm{AlGaN}$ growth leads to pronounced potential fluctuation and band-tail states, which contributes to carrier localization and enhance radiative recombination.

Figure 11 shows the normalized electroluminescence (EL) spectra for a number of UV LEDs grown in our laboratory by PAMBE. ${ }^{[114]}$

Figure 12 shows the performance characteristics of an unpackaged device emitting at $273 \mathrm{~nm} .{ }^{[113]}$ Figure 11 shows the electroluminescence (EL) spectra under pulsed injection up to $100 \mathrm{~mA}$. The inset of Figure 11 shows the integrated optical power output and EQE under both DC and pulsed injection. Heating limits the performance of the device who's EQE under DC injection is $0.4 \%$. The low EQE is partly due to heating and partly due to low light extraction of about $1 \%-2 \%$. To increase the light extraction it will be required to remove the sapphire substrate and texture appropriately the nitride film.

\section{UV lasers}

As discussed earlier several groups have reported the development of prototype optically pumped AlGaN-based deep UV lasers as well as demonstrated stimulated emission. ${ }^{[12-19]}$ In

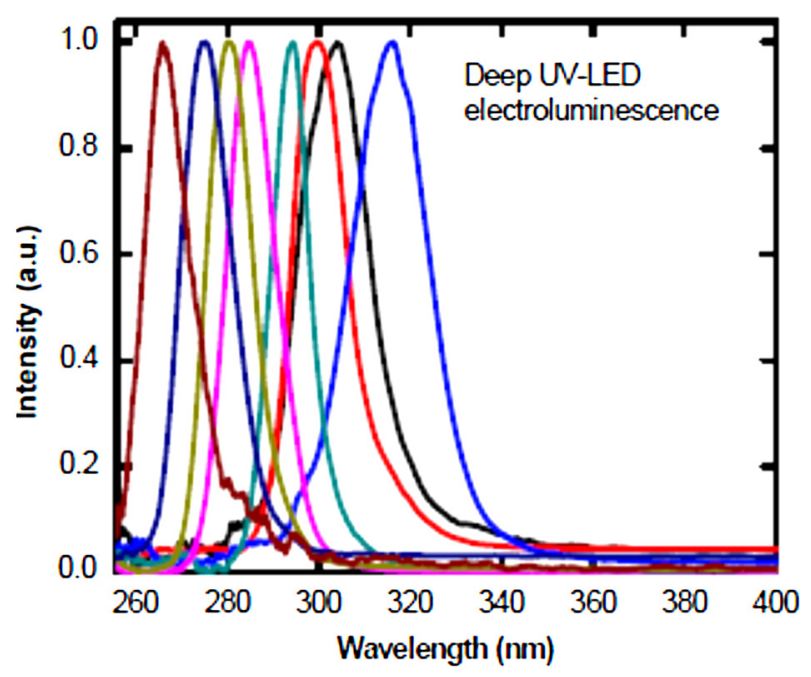

Figure 11. Normalized EL spectra of UV-LEDs emitting from 320 to $265 \mathrm{~nm}$, produced by PAMBE. ${ }^{[114]}$ 


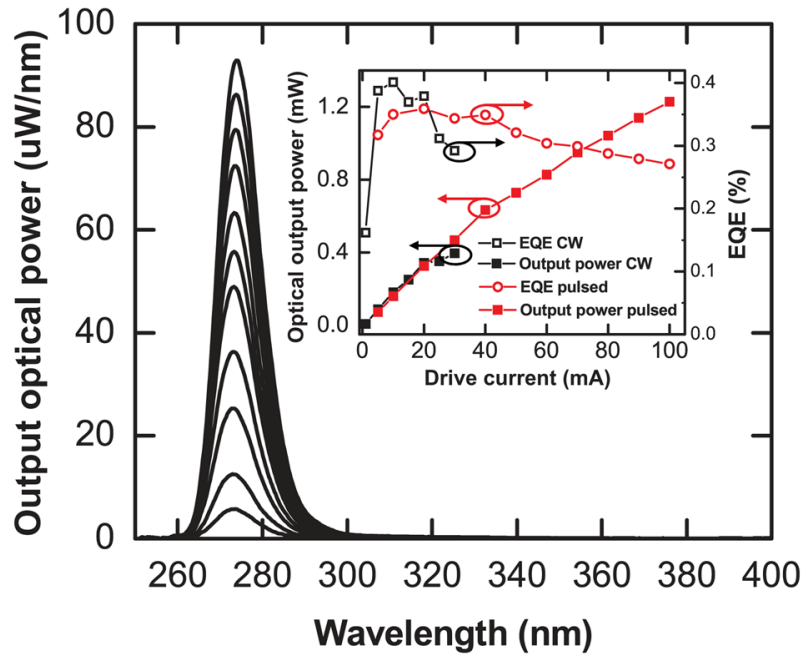

Figure 12. EL spectra of a UV-LED emitting at $273 \mathrm{~nm}$ measured under pulsed injection at $10 \%$ duty cycle. Inset shows the integrated optical power output under DC and pulsed injection as well as the calculated EQE of the device. ${ }^{[113]}$

2004, Takano et al. reported the first evidence of lasing at $241.5 \mathrm{~nm}$ under pulsed optical pumping with a threshold pumping power approximately $1200 \mathrm{~kW} / \mathrm{cm}^{2}$ at room temperature. ${ }^{[12]}$ In 2010, Jmerik et al. reported the first MBE grown device lasing at $303 \mathrm{~nm} .{ }^{[13]}$ After that a number of groups reported optically pumped lasers fabricated on AIN substrates with relatively lower threshold power density. ${ }^{[14,17]}$

The employment of MQW structures is one of the most effective approaches for obtaining lasing. However, homogeneous QWs require high-carrier density to invert their population before any stimulated emission process sets in. As discussed previously, our group has developed by PAMBE AlGaN/AlN MQWs emitting below $250 \mathrm{~nm}$ with high IQE on both sapphire and $\mathrm{SiC}$ substrates.

For evaluating the optical gain of these MQWs Pecora et al. investigated two different $\mathrm{AlGaN}$ MQW structures grown on the $\mathrm{Si}$ face of $6 \mathrm{H}-\mathrm{SiC}$ substrates. ${ }^{[16,18]}$ A schematic of the two samples is shown in Figure 13. In both structures, first

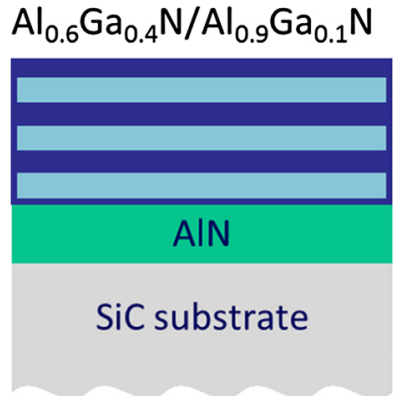

(a)

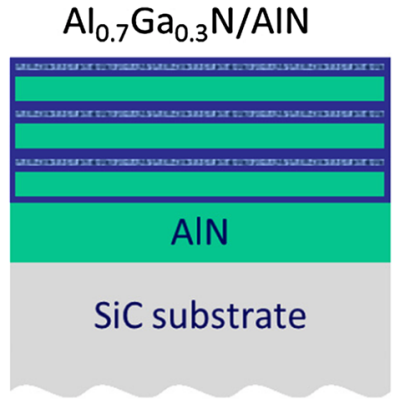

(b)
Figure 13. A schematic of the investigated AIGaN MQW samples. ${ }^{[18]}$ a 500-nm-thick AlN cladding layer was grown followed 10 periods of AlGaN MQWs consisting of $1.5 \mathrm{~nm}$ wells and $40 \mathrm{~nm}$ barriers. The first MQW (sample A), shown in Figure 13(a), consists of $\mathrm{Al}_{0.6} \mathrm{Ga}_{0.4} \mathrm{~N}$ wells and $\mathrm{Al}_{0.9} \mathrm{Ga}_{0.1} \mathrm{~N}$ barriers, while the second MQW, shown in Figure 13(b) consists of $\mathrm{Al}_{0.7} \mathrm{Ga}_{0.3} \mathrm{~N}$ wells and AlN barriers (sample $\mathrm{B}$ ). The AlGaN QWs in both samples were grown under Ga-rich conditions. During growth of sample A, a flux of indium has also been employed. Both structures were capped with a 100-nm-thick AlN layer for wave guiding. The number of QWs was selected to optimize the tradeoff between vertical optical confinement and material gain for fixed number of injected carriers. ${ }^{[122]}$

The emission properties of these structures were investigated using cathodoluminescence (CL) spectroscopy and mapping. Figure 14 shows the CL spectra and monochromatic CL maps for the two samples described in Figure 13. The striking difference between the two samples is that the sample, grown under Ga-rich conditions but in addition an indium flux, has a spatially homogeneous emission, while the sample grown only under Ga-rich conditions the monochromatic CL map reveals spatial non-uniformities on a submicron scale, consistent with the previously discussed formation of clustering and band-structure potential fluctuations in $\mathrm{AlGaN}$ alloys grown by the discussed method.

The microstructure of the sample B was investigated by TEM. ${ }^{[16]}$ High-angle annular-dark-field images were recorded with a probe size of $\sim 0.2 \mathrm{~nm}$, using a JEOL JEM-2010F operated in scanning TEM mode at $200 \mathrm{kV}$. Figure 15 shows a high-angle annular-dark-field ( $Z$-contrast) electron micrograph cross-section of the same sample. The enlarged image reveals nanocluster-like features within the $\mathrm{AlGaN}$ layer. The typical size of the nanoclusters in this image is approximately $2 \mathrm{~nm}$ in the plane and $1.5 \mathrm{~nm}$ (the well thickness) in the growth direction, suggesting QD behavior. However, given the highmagnification (i.e., small spatial extent) of this micrograph and the longer-range inhomogeneities observed in Figure 14, we are unable to estimate the actual density and average size of these nanoclusters. Further structural studies will, therefore, be required to fully characterize the possible role of 3D quantum confinement in these samples.

The optical gain properties of these MQWs were investigated and reported in a series of papers by Pecora et al. ${ }^{[16,18,123]}$ The variable-stripe length (VSL) methodology has been used for a detailed quantification of the gain properties. ${ }^{[124-128]}$ The reader is referred to the original papers by Pecora et al. for the details of the measurements and data analysis. In here we only present a summary of these data.

Deep-UV, $150 \mathrm{fs}$ laser pulses at $220 \mathrm{~nm}$ were used to optically pump the MQW structures. The pump laser is focused on the sample surface through a cylindrical lens forming a stripe whose length can be monitored and adjusted through a blade mounted in a motorized computer-controlled stage. The beam profile along the stripe has been measured through the knife-edge technique, resulting in a height of the stripe of 5 $\mu \mathrm{m}$ and a maximum stripe length of $250 \mu \mathrm{m}$, which provides 

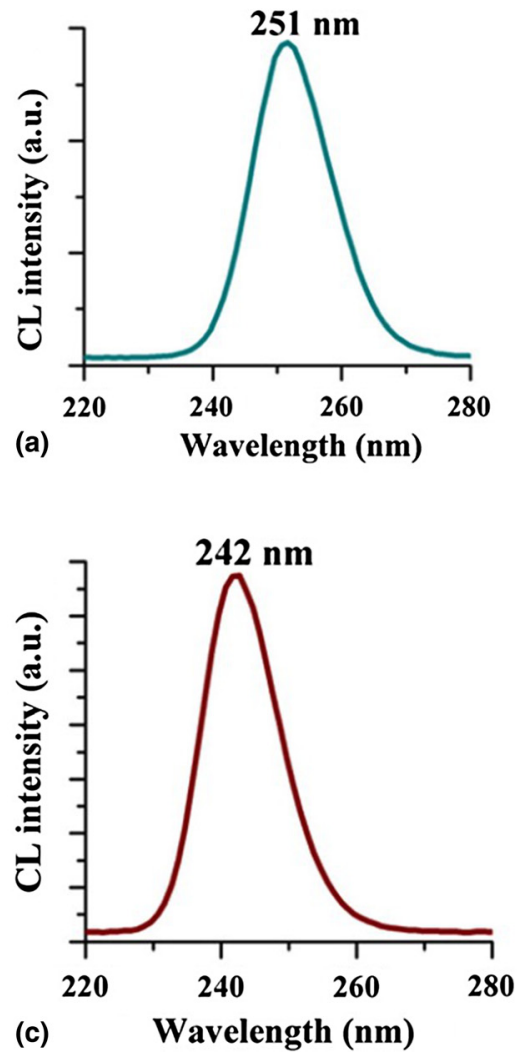

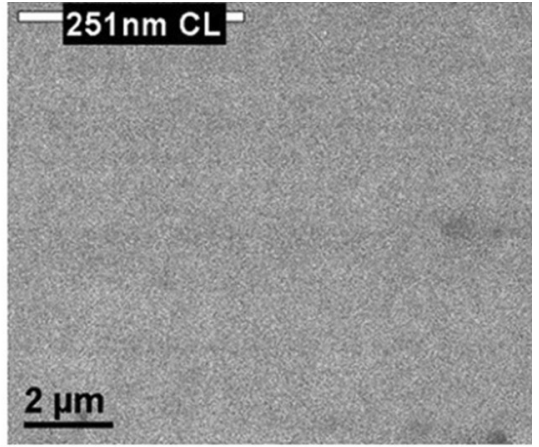

(b)

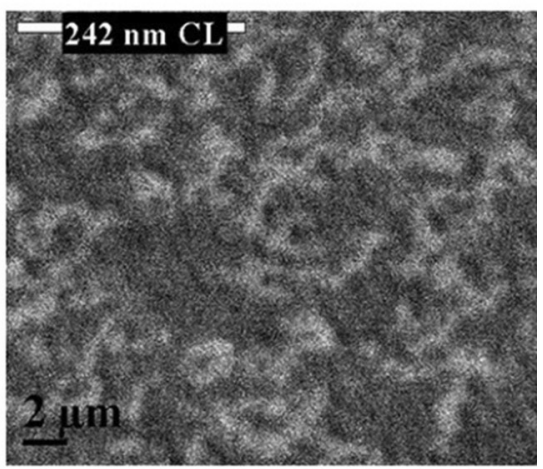

(d)

Figure 14. CL spectra and corresponding monochromatic CL maps for the two samples described in Figure 12. ${ }^{[18]}$

a homogeneous illumination of the sample. In the VSL setup, the samples were characterized by exciting the top surface and collecting the amplified spontaneous emission from the cleaved edge of the sample. ${ }^{[123]}$

The edge emission of the two samples, discussed in Figures 13 and 14, as a function of the pump fluence is shown in Figure 16. The measured spectrum intensity is divided by the excitation fluence in order better emphasize the nonlinear behavior of the emission. The spectra in Figure 16(a) correspond to sample A. The emission from this sample is sub-linear with the fluence, demonstrating absorption in this structure. On the other hand, the spectra in Figure 16(b), which correspond to the sample B, have the opposite behavior. As the pump fluence increases the signal intensity strongly increases with a clear superlinear trend demonstrating optical gain in this structure.

The spectra of Figure 16(b) were fitted as single Gaussian functions to determine the peak position and the full-width half-maximum (FWHM) of the emission and the data are plotted as a function of pumping fluence in Figure 17. Black dots refer to the left side axis, which reports the energy corresponding to the peak position. Red squares are relative to the right axis, which represents the FWHM of the spectra. As the pump fluence increases, the edge emission narrows and blueshifts. In particular, the FWHM decreases from 12.5 to 8.5 nm, while the peak position shifts up from 5.00 to $5.09 \mathrm{eV}$. The superlinear emission along with the blue shift and the spectral narrowing of this sample are strongly supporting the onset of stimulated emission in this sample.

On the other hand, we have conducted a similar analysis on the sample grown in the presence of indium, and found no changes in the peak position and the spectral width. This is consistent with the observed sub-linear trend of the measured edge emission with the pump fluence. As discussed earlier indium was found to be a surfactant during growth of $\mathrm{AlGaN}$ under slightly nitrogen-rich conditions of growth. ${ }^{[54]}$ On the other hand, this sample was grown under Ga-rich conditions. One possibility is that the Indium dissolves in the liquid $\mathrm{Ga}$ in the surface of the growing AlGaN film and forms $\mathrm{Ga}-\mathrm{In}$ liquid solution, ${ }^{[129]}$ which wets better the AlGaN seed than pure liquid $\mathrm{Ga}$ and forms a uniform in thickness $\mathrm{Ga}$-In liquid film covering the $\mathrm{AlGaN}$ seed. Thus, $\mathrm{Al}$ and active nitrogen dissolving into the liquid Ga-In film lead to laterally homogeneous AlGaN film.

Pecora et al. have also investigated the polarization properties of the edge emission of both samples in order to better understand the origin of the observed luminescence and gain. ${ }^{[123]}$ The polar plot of Figure 18, shows the peak intensity measured for both samples as a function of the analyzer angle. Red square 


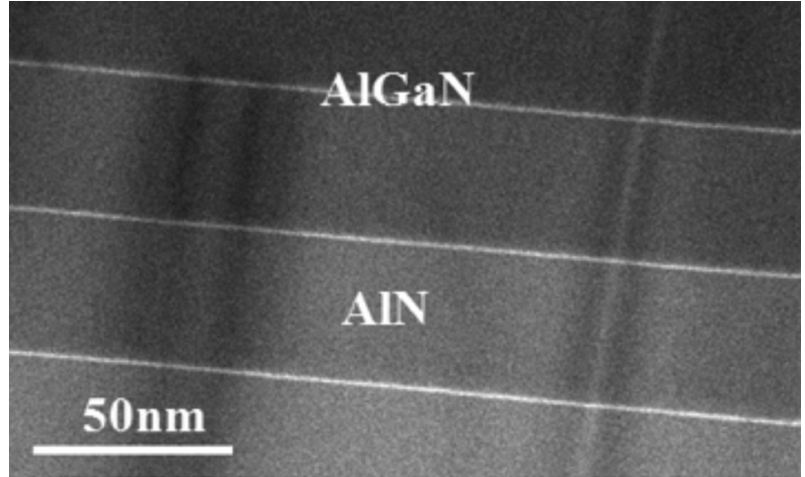

(a)

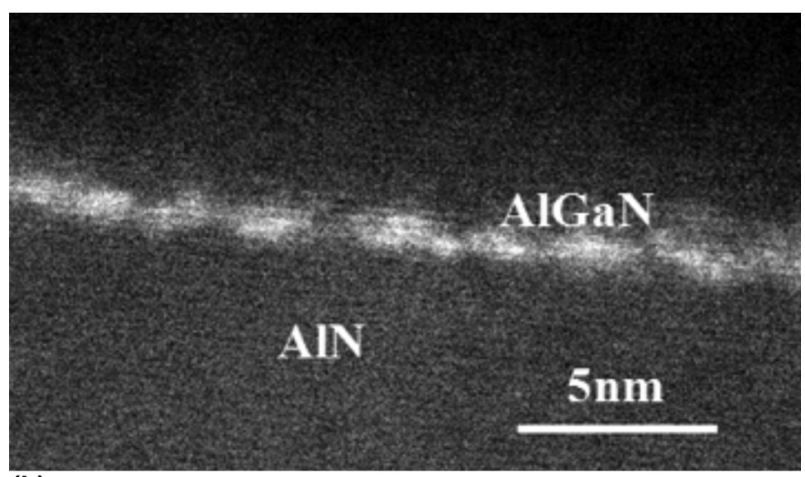

(b)

Figure 15. (a) High-angle annular-dark-field (Z-contrast) electron micrograph showing cross-section of AIN/AIGaN sample, and (b) enlarged image revealing cluster-like features within AIGaN layer. ${ }^{[16]}$

dots correspond to the structure A, while blue circles are relative to the structure $\mathrm{B} .0^{\circ}$ corresponds to the transversemagnetic (TM) and $90^{\circ}$ to the transverse-electric (TE) polarizations. Intensities have been recorded at the highest pump fluence $\left(60 \mu \mathrm{J} / \mathrm{cm}^{2}\right)$ and they are reported in a linear scale. First, we observe that the sample with compositional fluctuations is about a factor of five brighter than the other. More importantly, the emission from the homogeneous wells is totally unpolarized, while the compositional fluctuations introduce a different band order resulting in strongly TE polarized ASE.

The Al content in this sample is sufficiently high (70\%), and a turnover from the TE to the TM polarization is expected for an Al-content of $60 \%-80 \%$, depending on the thickness of the well and on the strain in the active layer. ${ }^{[130-133]}$ Since the wells in our structure are very thin, it is reasonable that the TE polarization is still predominant.

Using the VSL method Pecora et al. ${ }^{[18]}$ have also determined the gain coefficient from the evolution of the peakemission intensity as a function of the optically pumped sample length and from these data they obtained the absorption/gain spectrum for the two samples and the data are shown in Figure 19. Red squares correspond to the structure A. The spectrum appears to be featureless and always negative in values, as expected for an absorbing 1D waveguide, and it represents a

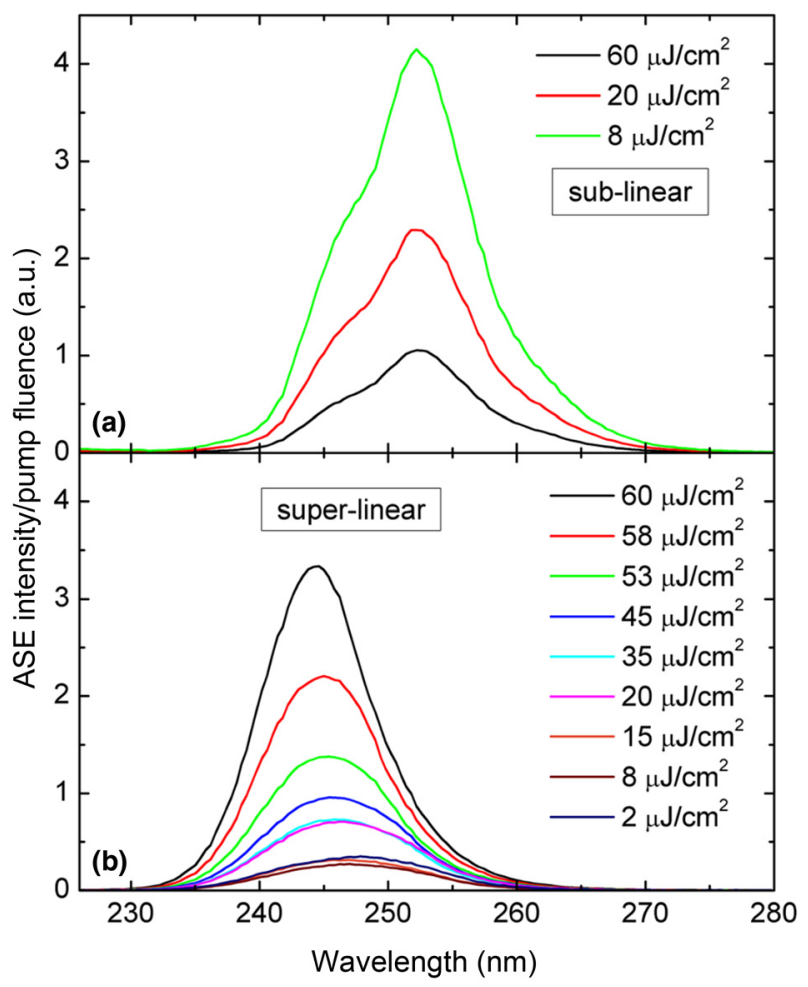

Figure 16. Edge emission as a function of the wavelength for the sample with homogeneous wells (a) and with strong band-structure compositional fluctuations (b). Data are scaled by the excitation fluence. ${ }^{[18]}$

measure of the net modal absorption coefficient of the material. The introduction of compositional fluctuations in the samples dramatically modifies the gain spectrum. Blue circles are mostly in the positive side of the graph, indicating that the sample is driven well in the amplification regime. On the longer

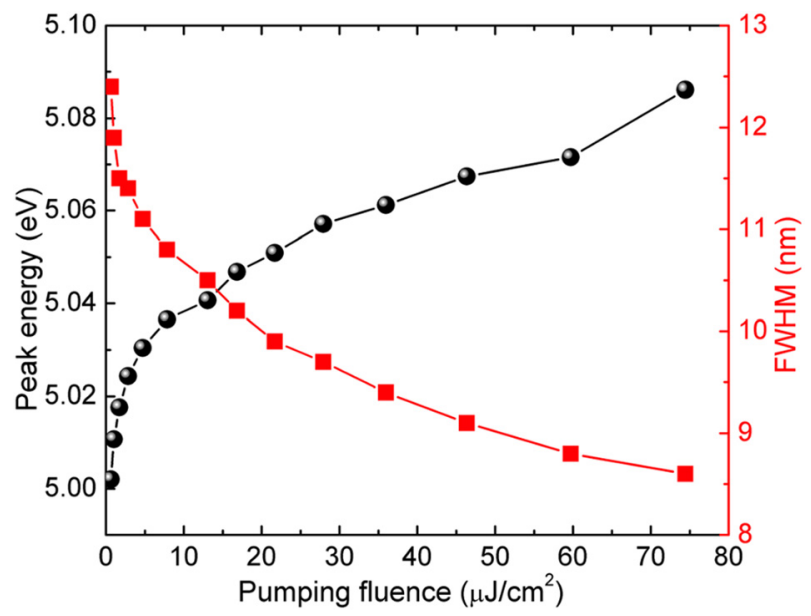

Figure 17. Peak position (black dots, left-side axis) and FHWM (red circles, right-side axis) of the edge emission spectra from the sample with compositional fluctuations as a function of the pump fluence. ${ }^{[18]}$ 


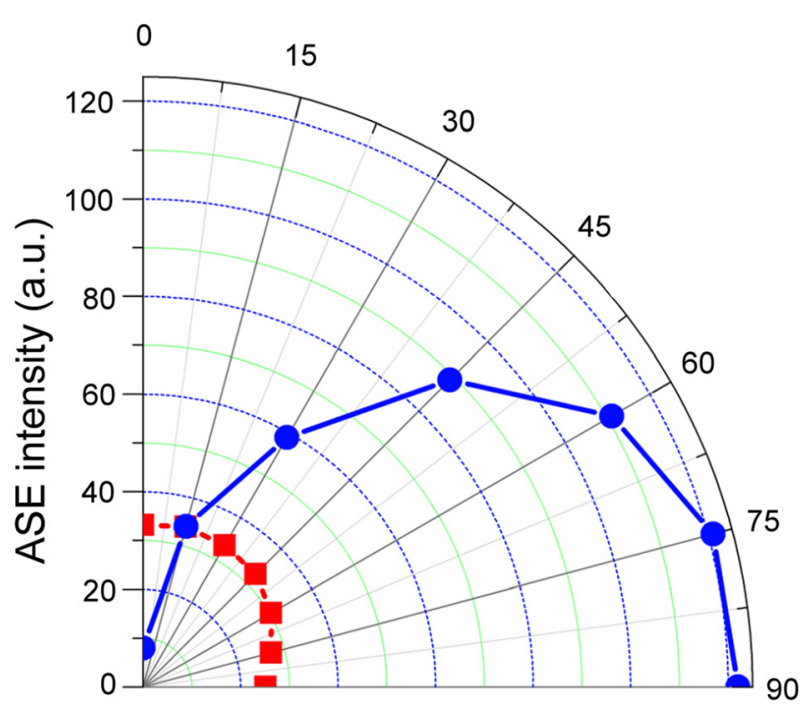

Figure 18. Measured peak intensity for the sample with (black dots) and without (red circles) compositional fluctuations as a function of the analyzer angle. ${ }^{[18]}$

wavelength side of the spectrum, the measured data turn into negative values. The wavelength of zero gain is at $266 \mathrm{~nm}$ $(4.66 \mathrm{eV})$, which represents an experimental estimation of the effective band gap of the material. We notice that this effective gap value should not be confused with the estimated gap of a homogeneous material for the nominal Al content.

The data of Figure 19 clearly demonstrate optical gain in AlGaN MQWs with band-structure potential fluctuations down to $230 \mathrm{~nm}$ with a maximum net modal gain value of $120 \mathrm{~cm}^{-1}$. The optical gain threshold was measured to be 5 $\mu \mathrm{J} / \mathrm{cm}^{2}$ from which we estimate the density of optically excited

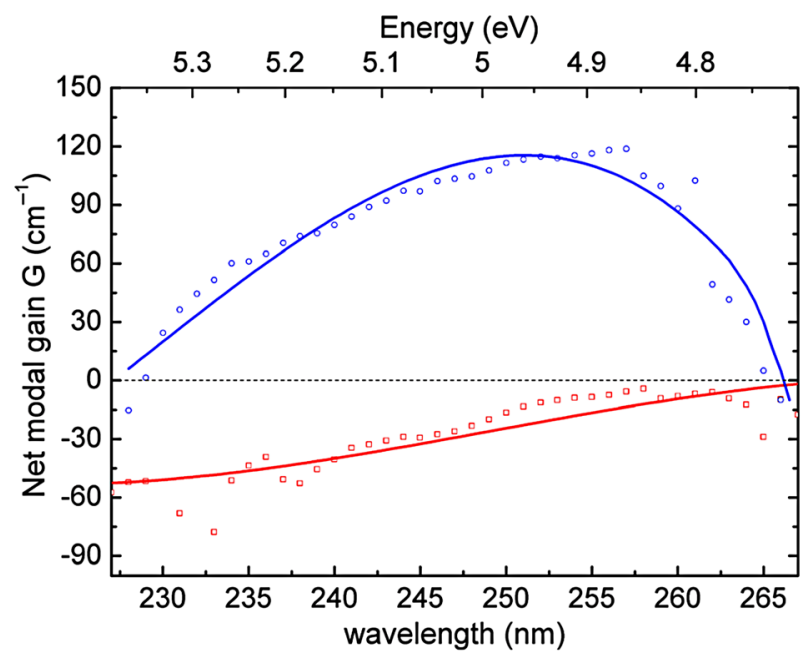

Figure 19. Absorption/gain spectra measured at the highest pump fluence for the sample with (blue dots) and without (red circles) compositional fluctuations. ${ }^{[18]}$ carriers at the threshold to be $1.4 \times 10^{17} \mathrm{~cm}^{-3}$. This is two orders of magnitude lower than what can be achieved with homogeneous QW structures, ${ }^{[18]}$ which highlights the benefits of introducing band-structure potential fluctuations in the MQW layers for laser applications.

Recently, our group has also proposed the fabrication of deep-UV lasers based on AlGaN alloys in the form of a graded-index separate confinement heterostructure (GRINSCH), as schematically shown in Figure 20. ${ }^{[129,134-136]}$ Such laser device structures were successfully used in traditional III-V compounds and were found to have the lowest threshold current. Besides the efficient carrier and optical field confinement in lasers based on the GRINSCH configuration, such laser designs based on AlGaN alloys have the additional advantage of automatically leading to a $\mathrm{p}-\mathrm{n}$ junction formation, ${ }^{[134]}$ owing to opposite compositional grading of the AlGaN alloys in either side of the active region of the device. ${ }^{[137]}$ Thus, such an $\mathrm{AlGaN}$ laser structure has the potential to overcome the difficulties associated with the efficient doping of AlGaN alloys. Stimulated emission and optical gain in these devices was also demonstrated. ${ }^{[136]}$ The crystal microstructure and optical properties of such GRINSCH devices can be found in the original papers. ${ }^{[129,134-136]}$

\section{Photodetectors}

The extremely strong absorption and radiation-induced aging effects in most semiconductor materials has historically hampered the development of high-quantum-efficiency semiconductor UV detectors. AlGaN-based UV detectors, unlike those based on traditional semiconductors such as silicon and gallium arsenide, are making strides in detecting UV radiation-from $400 \mathrm{~nm}$ to x-rays, as well as alpha particles-with improved sensitivity, high spectral selectivity, and low noise. With these advances, nitride-based UV detectors are finding use in areas such as the detection of UV flames for combustion control, surveillance of rockets and intercontinental ballistic

\begin{tabular}{|c|}
\hline $100 \mathrm{~nm}$ AlN cladding layer \\
\hline $50 \mathrm{~nm}$ AlGaN graded up \\
\hline $75 \mathrm{~nm}$ Bulk $\mathrm{Al}_{0.72} \mathrm{Ga}_{0.28} \mathrm{~N}$ \\
\hline $50 \mathrm{~nm}$ AlGaN graded down \\
\hline $500 \mathrm{~nm}$ AlN cladding and buffer layer \\
\hline (0001)6H-SiC
\end{tabular}

Figure 20. Schematic representation of the investigated GRINSCH double heterostructure. ${ }^{[134]}$ 
missiles, secure space-to-space communication, detection of UV scintillation for medical imaging, monitoring of pollutants such as nitrous oxide and sulfur dioxide in the ionosphere, in space-based instrumentation for UV astronomy and in UV photolithography for semiconductor processing. As shown in Figure 21, the AlGaN-based material system is well suited for UV photodetectors because its direct band gap can be tuned from 360 to $200 \mathrm{~nm}$ by changing the alloy composition, enabling true visible-blind or solar-blind detectors. ${ }^{[21-29]}$ Excellent reviews on UV photodetectors were presented in Refs 23, 26.

Semiconductor UV detectors can be made to operate either in the photoconductive or the photovoltaic mode. The photovoltaic detectors can have the form of a Schottky barrier, $\mathrm{p}-\mathrm{i}-\mathrm{n}$ diode, avalanche photodiode, heterojunction phototransistor, or charge-coupled devices. ${ }^{[23,26]}$

Photoconductive detectors are fabricated from AlGaN alloys in thin-film form with interdigitated metal contacts placed on the surface of the material to maximize light transmission while minimizing transit time. These photoconductive detectors, as opposed to photovoltaic ones, exhibit the important advantage of internal gain, which reduces the requirement for low-noise preamplifiers. The gain is due to the fact that the minority carriers are trapped while the majority carriers go around the circuit many times before recombination. Of course the fundamental principle in all detectors is that the gain times the bandwidth should be constant. Thus, if the detector is designed to have high photoconductive gain, its response time is long. The photoconductive gain is given by the expression:

$$
G=\mu \tau V / d^{2}=\tau / t_{\mathrm{r}}
$$

Here, $\mu$ is the electron mobility, $\tau$ is the carrier lifetime, $V$ is the applied bias voltage, $d$ is the inter-electrode spacing, and $t_{\mathrm{r}}$ is the carrier transit time. Thus, the gain in photoconductive detectors occurs because the recombination lifetime is much

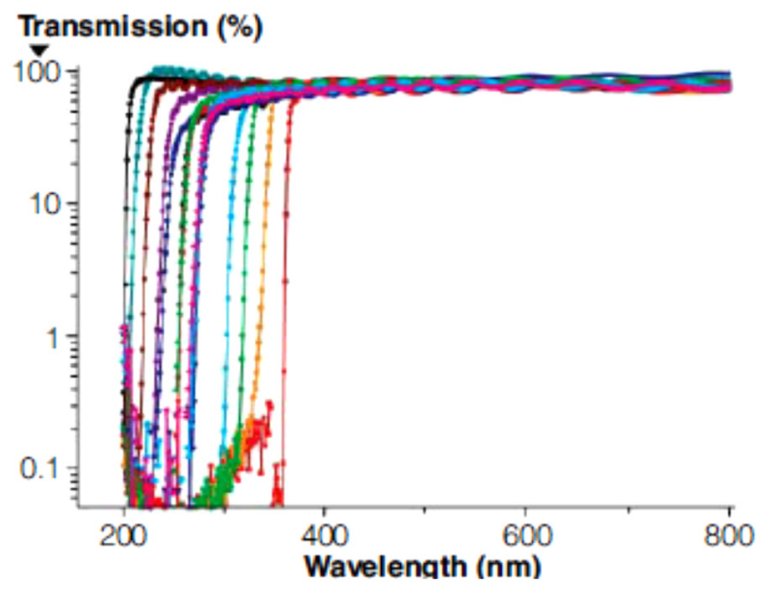

Figure 21. The optical transmission spectra of $\mathrm{Al}_{x} \mathrm{Ga}_{1-x} \mathrm{~N}$ thin films as a function of composition $x^{[29]}$ longer than the transit time. As a result, while the gain depends on the dimensions of the device and the applied voltage, the $(\mu \tau)$ product-determined from the gain measurements-is the real figure of merit of the material.

The values of the photoconductive gain and responsivity of AlGaN UV detectors, reported by various groups, were found to vary widely. ${ }^{[21-29]}$ To find the origin of this variation, we fabricated a number of UV photoconductive detectors from $\mathrm{GaN}$ of varying resistivity and $\mathrm{AlGaN}$ of various compositions and determined the $(\mu \tau)$ product from the gain measurements. ${ }^{[25,28,138]}$ Figure 22 shows the $(\mu \tau)$ product for the fabricated $\mathrm{GaN}$ and $\mathrm{AlGaN}$ photoconductive detectors. ${ }^{[28]}$ It is evident from these data that the $(\mu \tau)$ product in these photodetectors varies by many orders of magnitude and is a strong function of resistivity of the semiconductor. Thus, by using $\mathrm{GaN}$ or $\mathrm{AlGaN}$, UV detectors can be fabricated with either high gain/low speed, or vice versa. ${ }^{[28]}$

The data in Figure 22 indicate that AlGaN photodetectors have higher $(\mu \tau)$ products than GaN ones, even though they are expected to have lower mobility than GaN because of alloy scattering and shorter lifetime than GaN because of their more defective nature. To account for this anomaly our group investigated the structure of the AlGaN alloys and found that these materials consist of domains that are atomically ordered and others that have random alloy structure (partial ordering ordering). ${ }^{[79]}$ Based on this finding we have proposed $^{[25]}$ that the band-structure of the ordered and random domains form a type-II heterostructure as shown in Figure 23. Thus, the electron-hole pairs created by the illumination are separated in the ordered and random domains, which lead to the observed enhancement of the lifetime. This hypothesis was later supported by atomistic empirical pseudopotential simulations reported by Dudiy and Zunger. ${ }^{[139]}$ These authors found that the band alignment between random and ordered

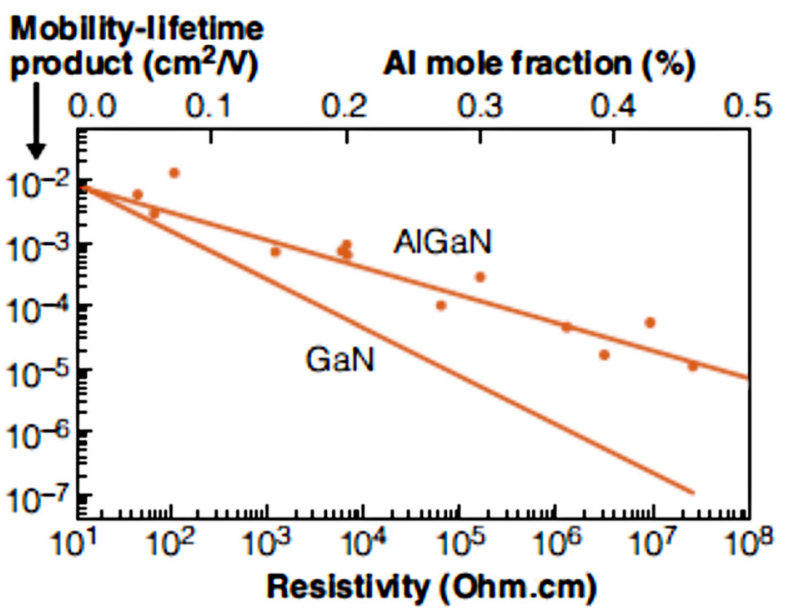

Figure 22. The mobility-lifetime product $(\mu \tau)$ in $\mathrm{Al}_{x} \mathrm{Ga}_{1-x} \mathrm{~N}$ and GaN photodetectors varies with film resistivity. ${ }^{[25,28]}$ 

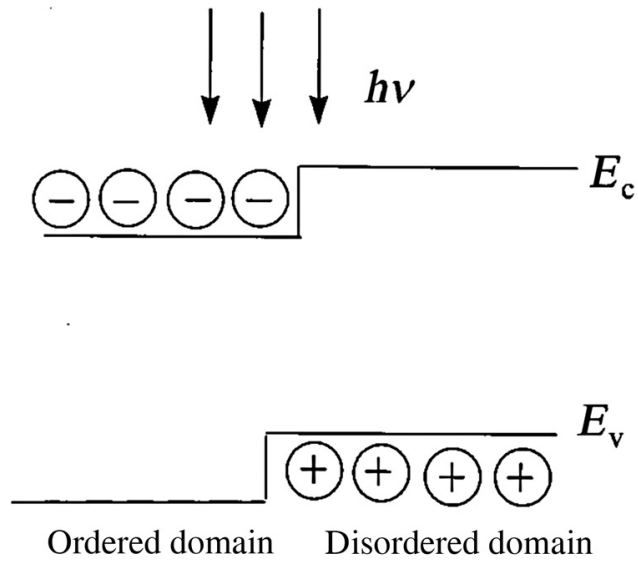

Figure 23. Schematic illustration of the potential band alignment of ordered and disordered domains in the $\mathrm{Al}_{x} \mathrm{Ga}_{1-x} \mathrm{~N}$ alloys. ${ }^{[25,28]}$

domains changes from types I to II at about $40 \%$ AlN mole fraction in the AlGaN alloys.

\section{Optical modulators}

Electroabsorption modulators are semiconductor electro-optic devices in which a change in absorption coefficient is induced by an externally applied electric field. Such devices based on cubic III-V semiconductors have been the subject of extensive research over the past three decades and have found a variety of applications in, e.g., fiber-optic data transmission, photonic switching, and optical interconnects. In general, particularly strong modulation can be obtained when the absorption edge is dominated by excitonic effects, due to the sharp nature of the resulting absorption features.

However, in smaller-band gap bulk semiconductors such as $\mathrm{GaAs}$ the room temperature exciton-binding energy ( $\sim 4 \mathrm{meV})$ is substantially less than the thermal energy $k_{\mathrm{B}} T$ at room temperature. As a result, the excitonic nature of the absorption edge at room temperature is not obvious due to thermal broadening, and the associated benefits for electroabsorption modulation are lost. On the other hand, the exciton-binding energy becomes substantially larger in QW structures, leading to wellresolved excitonic absorption peaks even at room temperature. High-performance optical modulators have therefore been developed over the years based on the quantum confined Stark effect in QWs, ${ }^{[140]}$ where large changes in the excitonic resonance are obtained through the application of an electric field along the growth direction.

Research in the area of electroabsorption modulators based on wurtzite III-nitride semiconductors is still in the very early stage of development. ${ }^{[30-36]}$ In these materials, strong electric fields are already present in the QWs due to spontaneous and piezoelectric polarizations; as a result, an even greater change in absorption is achievable, especially if the internal fields are compensated by the external bias so that the net field in the QWs is reduced. Such devices are likely to find a number of applications. Of particular interest is their development for non-line-of-sight free-space optical communications based on atmospheric light scattering where the use of short-wavelength radiation is advantageous due to its large scattering crosssection. External optical modulators in these systems would allow for higher transmission rates, without the deleterious transient heating effects that are typically associated with direct current modulation of semiconductor light sources. Nitride electroabsorption modulators, incorporated within a laser cavity, have also been used for the generation of short pulses of visible/UV radiation via $Q$-switching. ${ }^{[30]}$

A basic property of nitride semiconductors that is particularly important in this context is provided by their large exciton-binding energies (about $25 \mathrm{meV}$ in $\mathrm{GaN}$ and even higher in ternary AlGaN alloys). This is a direct consequence of the heavy electron and hole effective masses of these materials, which in turn are directly related to their large band gap energies. As a result, even in bulk samples at room temperature the optical absorption edge is dominated by excitonic effects, so that strong electroabsorption of near band gap radiation can be expected.

\section{Electroabsorption modulators based on bulk GaN films}

A UV optical modulator based on a $0.4-\mu \mathrm{m}$-thick $\mathrm{GaN}$ film grown by MOCVD has been reported by Oberhofer et al. ${ }^{[31]}$ However, this device was found to require a prohibitively large applied voltage $(>80 \mathrm{~V})$ to produce any appreciable change in transmission. Specifically a maximum modulation depth under normal-incidence operation of $18 \%$ at $305 \mathrm{~V}$ bias was reported.

Our group reported ${ }^{[35]}$ the development of a GaN electroabsorption modulator grown by RF PAMBE with similar active layer thickness and device geometry with dramatically improved performance over that reported in Ref. 31 A schematic of the investigated device is shown in Figure 24. Following nitridation of the sapphire surface, a relatively thick $(0.5 \mu \mathrm{m})$ AlN film was initially grown in this structure, so that all subsequent epitaxial layers are under compressive strain which

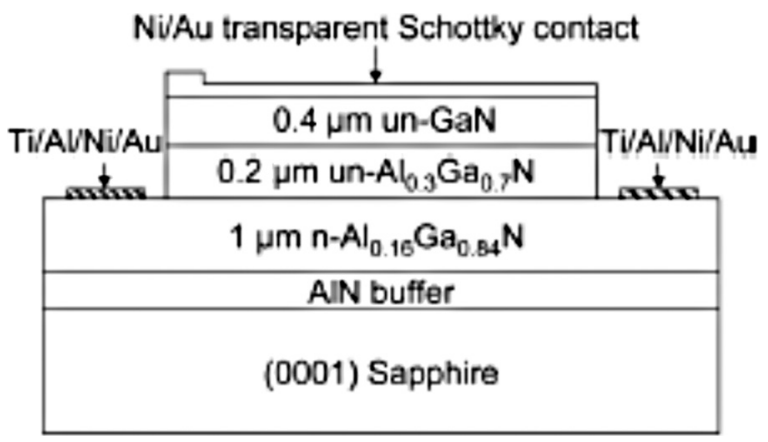

Figure 24. Schematic cross-sectional view of the bulk GaN optical modulator. ${ }^{[35]}$ 
reduces their probability of developing cracks. A transparent contact layer consisting of Si-doped $\mathrm{n}-\mathrm{Al}_{0.16} \mathrm{Ga}_{0.84} \mathrm{~N}$ was then deposited, followed by a nominally intrinsic $\mathrm{Al}_{0.3} \mathrm{Ga}_{0.7} \mathrm{~N}$ film whose function is to electrically isolate the GaN active region from the bottom contact layer. The active region is also nominally undoped (with an estimated density of unintentional donor impurities of about $10^{17} \mathrm{~cm}^{-3}$ ) and has a nominal thickness of $0.4 \mu \mathrm{m}$.

The electroabsorption devices shown in Figure 24 were fabricated by standard photolithography and inductively coupled plasma etching in chlorine. Ohmic contacts to $\mathrm{n}-\mathrm{Al}_{0.16}$ $\mathrm{Ga}_{0.84} \mathrm{~N}$ were formed using the multilayer structure $\mathrm{Ti} / \mathrm{Al} / \mathrm{Ni} /$ $\mathrm{Au}$ and transparent Schottky contact was formed on the GaN active region using $\mathrm{Ni} / \mathrm{Au}$.

Prior to the device fabrication, the material optical absorption spectrum was determined via transmission measurements at room temperature and the results are plotted in Figure $25 .{ }^{[35]}$ These data clearly show that even without cryogenic cooling the absorption edge of this bulk sample is dominated by excitonic effects, leading to a sharp peak at a photon energy of about $3.47 \mathrm{eV}$. The abrupt increase in absorption at about $3.7 \mathrm{eV}$ is due to the $1-\mu \mathrm{m}$-thick $\mathrm{Al}_{0.16} \mathrm{Ga}_{0.84} \mathrm{~N}$ film.

The normal-incidence transmission spectra under different reverse bias conditions were measured and the data are shown in Figure 26. ${ }^{[35]}$ The measured transmission spectra at various reverse bias voltages from 0 to $14 \mathrm{~V}$ through the device were then normalized to similarly measured transmission spectra through a sapphire substrate. As the applied voltage is increased, the excitonic absorption resonance is broadened and quenched, leading to an increase in transmission near the exciton peak and to a decrease in transmission at sufficiently detuned photon energies. From these data we obtain a maximum modulation depth $M$ of about $30 \%$ at a photon energy of about $3.45 \mathrm{eV}$, where $M$ is defined as the ratio $[T(V)-T$ $(0)] / T(0)$ and $T(V)$ is the device transmission as a function of bias voltage $V$.

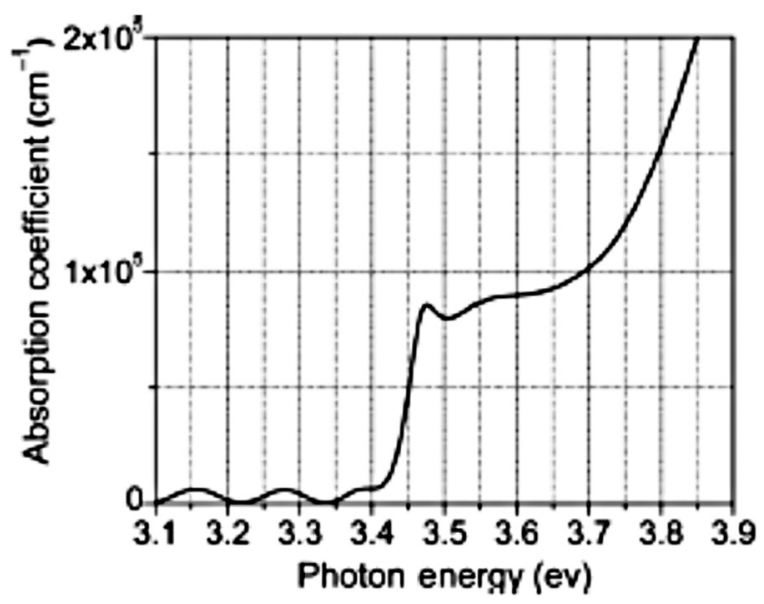

Figure 25. Absorption spectrum of the epitaxial material used to fabricate the device shown in Figure 24. ${ }^{[35]}$

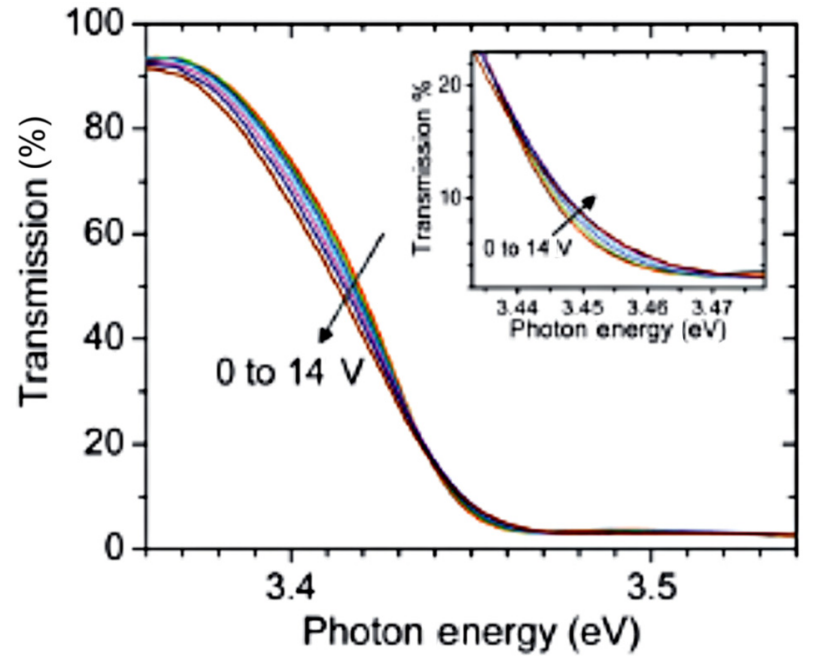

Figure 26. Normalized transmission spectra through a bulk GaN for different values of the applied reverse bias voltage from 0 to $14 \mathrm{~V}$ in steps of $2 \mathrm{~V}$. The inset shows a zoom-in of those traces near the excitonic resonance. ${ }^{[35]}$

The measured transmission spectra can also be used to calculate the corresponding changes in absorption coefficient $\Delta \alpha(V)=\alpha(V)-\alpha(0)$ versus photon energy, for different values of the applied voltage. Specifically, since $T(V)$ is proportional to $\exp [-\alpha(V) d]$, where $d$ is the thickness of the absorbing layer ( $0.4 \mu \mathrm{m}$ in this case), we can calculate $\Delta \alpha(V)$ from Eq. (7).

$$
\Delta \alpha(V)=-1 / d\{\ln [T(V) / T(0)]\}
$$

Several spectra of $\Delta \alpha$ obtained with this procedure are shown in Figure 27. ${ }^{[35]}$ The maximum change in absorption

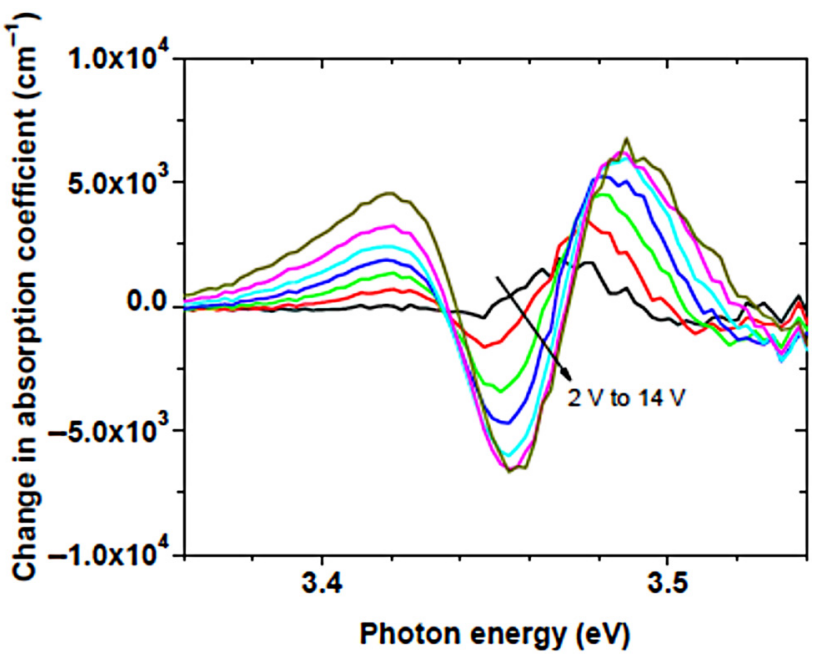

Figure 27. Differential absorption spectra for a bulk GaN modulator for different values of the applied reverse bias voltage from 0 to $14 \mathrm{~V}$ in steps of $2 \mathrm{~V} .^{[35]}$ 
coefficient here is found to increase with applied voltage up to a peak (absolute) value of about $7 \times 10^{3} \mathrm{~cm}^{-1}$ at $V=12 \mathrm{~V}$. As the reverse bias is further increased, a non-negligible amount of leakage current begins to flow across the active layer. This leads to a thermal modulation of the band edges via resistive heating, which tends to compensate the field-induced changes in the absorption edge. As a result, no further increase in $\Delta \alpha$ is obtained at higher voltages.

\section{Electroabsorption modulators based on GaN/AIGaN MQWs}

A UV optical modulator based on GaN/AlGaN MQWs, was first reported by Friel et al. ${ }^{[32]}$ This modulator structure was grown by RF PAMBE on (0001) sapphire and is shown schematically in Figure 28. The modulator active region consisted of 10 periods of $\mathrm{Al}_{0.17} \mathrm{Ga}_{0.83} \mathrm{~N} / \mathrm{GaN} \mathrm{QWs}$, nominally undoped. The well and barrier widths were designed to be 50 and $40 \AA$, respectively. Devices were fabricated using standard photolithographic techniques. Mesa structures were formed by inductively coupled plasma etching using chlorine. A Schottky contact was formed directly on top of the QWs using a thick $\mathrm{Pt} / \mathrm{Au}$ pad on one corner of the mesa and thin $\mathrm{Pt} / \mathrm{Au}$ semitransparent contact over the remainder of the mesa surface. The external field across the QWs was controlled by applying

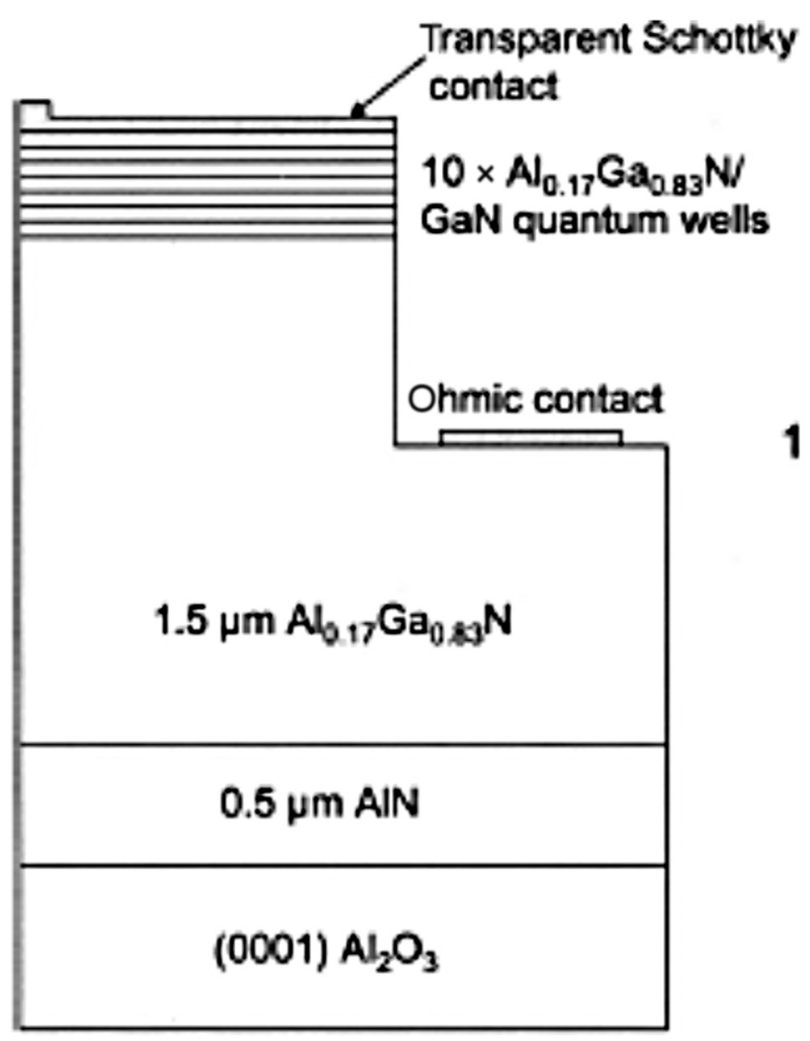

Figure 28. Schematic of a UV optical modulator structure based on GaN/ $\mathrm{Al}_{0.17} \mathrm{Ga}_{0.83} \mathrm{~N} \mathrm{MQWs}{ }^{[32]}$ a reverse bias to the Schottky contact. For group-III-polar materials this leads to a compensation of the internal fields in the QWs.

Figure 29 shows the optical transmission through the device as a function of reverse bias. The inset shows the transmission over an extended energy range. At photon energies below 3.4 $\mathrm{eV}$, thin-film interference fringes can be observed. The attenuation of light between about 3.4 and $3.7 \mathrm{eV}$ is due to absorption in the QWs and the absorption edge at about $3.75 \mathrm{eV}$ is due to the $\mathrm{Al}_{0.17} \mathrm{Ga}_{0.83} \mathrm{~N}$ :Si cladding layer. The observed changes in the transmission spectra with increasing reverse bias are attributed to enhancement of the excitonic absorption in the QWs.

The optical transmission data in Figure 29 were used to calculate the change in the absorption coefficient in the QWs $\Delta \alpha$ due to the applied bias. Since the transmission $T(V)$ at a bias voltage $V$ is proportional to $e^{-\alpha(V) d}$, where $d$ is the thickness of the absorbing layer (total thickness of the QW layers), $\Delta \alpha$ $(V)=\alpha(V)-\alpha(0)$, is given by Eq. (7) and plotted in Figure 30. A clear enhancement of the excitonic resonance with increasing reverse bias can be seen at about $3.48 \mathrm{eV}$.

Focusing on the main excitonic resonance at about $3.48 \mathrm{eV}$ in Figure 30, we note that the induced change in the absorption coefficient is around one order of magnitude larger than that of typical III-arsenide or III-arsenide-phosphide electroabsorption modulators, for comparable bias voltages, ${ }^{[140]}$ demonstrating the great potential for using these devices in non-linear optical applications. The observed order of magnitude increase in $\Delta \alpha$ $(V)$ in comparison to that of other III-V materials is simply a reflection of the greater near-band-edge absorption coefficient in III-nitrides of about $10^{5} \mathrm{~cm}^{-1}$ in relation to, for example, III-arsenides of about $10^{4} \mathrm{~cm}^{-1}$.

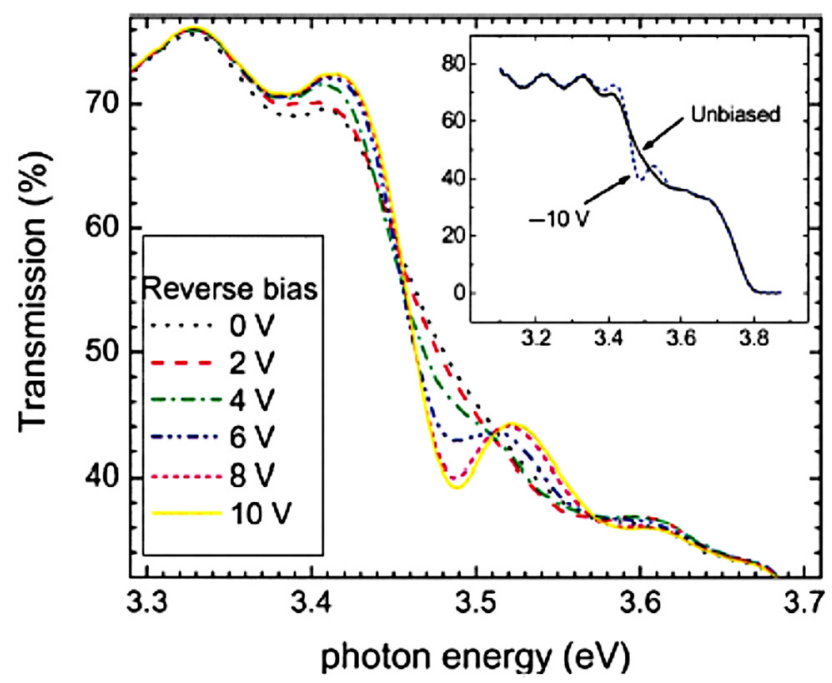

Figure 29. Optical transmission spectra through the modulator device as a function of applied reverse bias. The insert shows the spectra over an extended photon energy range for 0 and $-10 \mathrm{~V}$ bias. ${ }^{[32]}$ 


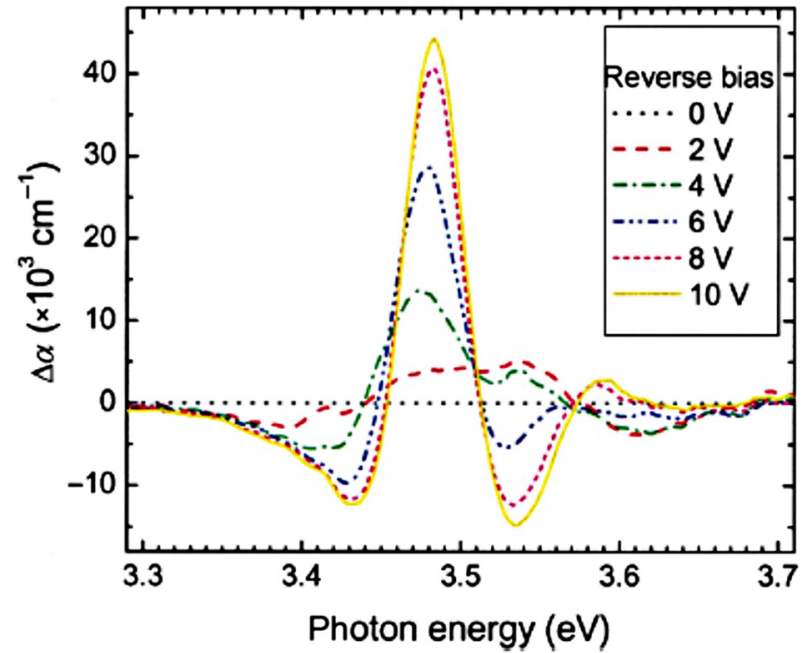

Figure 30. Change in the absorption coefficient of the optical modulator as a function of applied bias. ${ }^{[32]}$

\section{Distributed Bragg reflectors}

Our group also reported progress in developing DBRs based on AlGaN alloys by PAMBE. ${ }^{[141-145]}$ The efficiency of UV

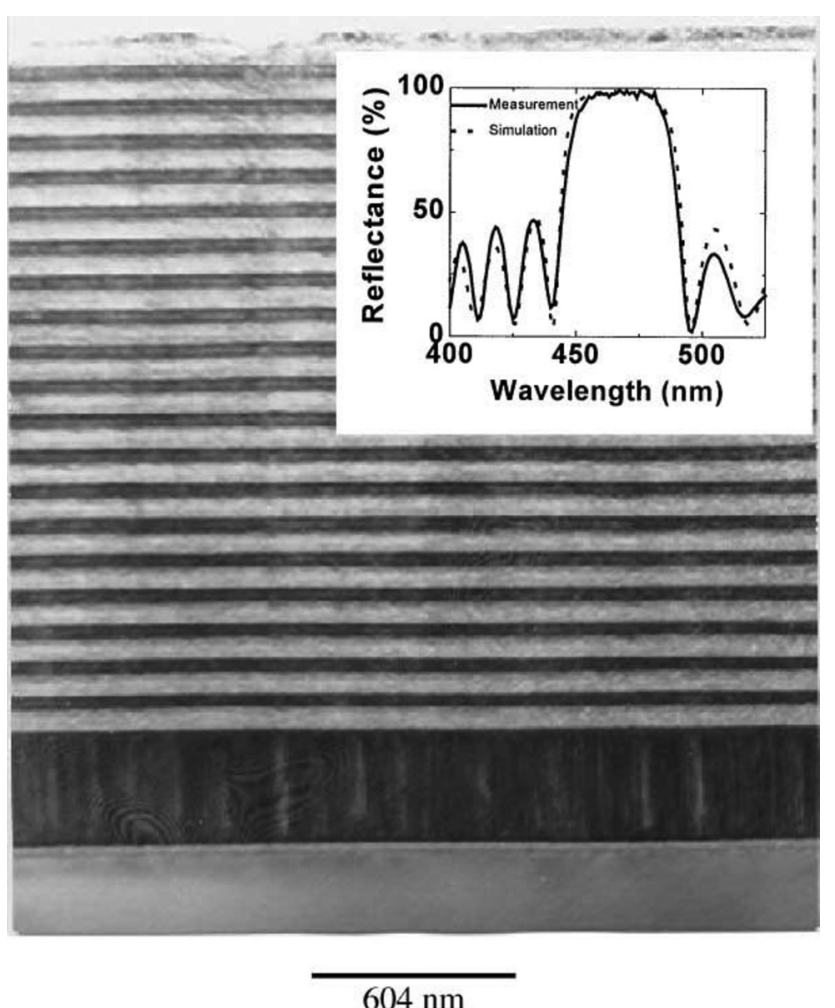

Figure 31. Cross-section TEM micrograph of a 20.5 period AIN/GaN DBR stack. Inset shows experimental reflectivity spectra from this DBR along with simulation results based on the transition matrix method. ${ }^{[153]}$

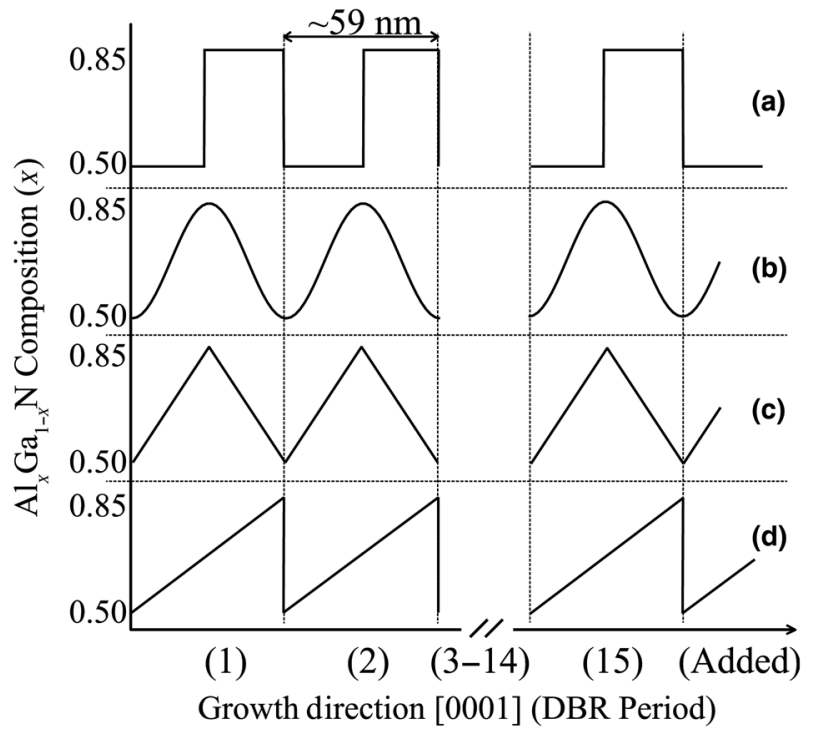

Figure 32. Schematics of investigated DBRs with (a) square, (b) sinusoidal, (c) triangular, and (d) sawtooth AIGaN grading profiles. Each DBR has 15 periods and is capped with an extra partial period for proper phasing. ${ }^{[145]}$

optoelectronic devices can improve significantly if they are designed in a resonant cavity form, such as resonant cavity LEDs, vertical-cavity surface-emitting lasers, resonant cavity detectors, and asymmetric Fabry-Perot electroabsorption modulators. ${ }^{[146]}$ Such resonant cavity devices require high reflectivity DBRs. Honda et al. have estimated the threshold current density in a GaN VCSEL structure and concluded that an increase in the peak reflectance of the DBR mirror from $90 \%$ to $99 \%$ results in more than an order of magnitude reduction in the threshold current density. ${ }^{[147]}$

Another important requirement for the fabrication of nitride resonant cavity devices is the large bandwidth of the primary reflectance peak. This is important because the active region of the nitride devices is based on alloy heterostructures or MQWs, whose optical properties are very sensitive to small variations in growth or process parameters. As we have discussed earlier AlGaN and also InGaN ${ }^{[148]}$ alloys can develop compositional inhomogeneities and various types of alloy ordering depending on growth parameters. Such phenomena affect their optical properties resulting in spectral shift of their absorption edge and emission spectra.

The main difficulty in fabricating nitride DBRs with high reflectivity and large bandwidth is the small index of refraction contrast that can be obtained within the entire AlGaN alloy composition. A number of groups have reported the fabrication of $\mathrm{AlGaN} / \mathrm{GaN}$ DBRs with peak reflectance in the near ultraviolet to blue-green region of the spectrum. ${ }^{[149-151]}$ With the employment of 30-40 quarter wave periods, peak reflectivities at $390 \mathrm{~nm}$ of $96 \%$ were obtained with a bandwidth of about $14 \mathrm{~nm} .{ }^{[150]}$ A similar device was reported to have peak reflectance of $96 \%$ at $460 \mathrm{~nm}$ with a bandwidth of $22 \mathrm{~nm} .^{[151]}$ 


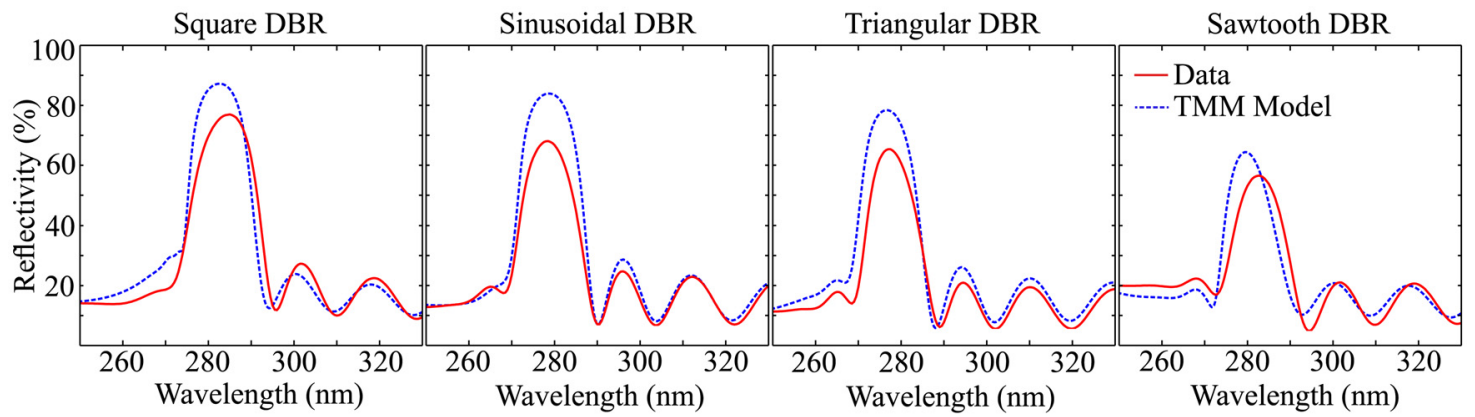

Figure 33. The measured (red) and modeled (dashed blue) reflectivity spectra for the square, sinusoidal, triangular, and sawtooth DBRs. ${ }^{[145]}$

DBRs based on AlN/GaN quarter wave stacks have the potential for higher peak reflectance and larger bandwidth with approximately half the number of quarter wave periods. Such DBRs were reported early on by our group using PAMBE ${ }^{[141-143]}$ and the Sandia group using gas source MBE. ${ }^{[152]}$ The cross-section TEM micrograph of a DBR stack consisting of 20.5 periods of $\mathrm{AlN} / \mathrm{GaN}$ with thickness of 62.3 and $40.3 \mathrm{~nm}$, respectively, is shown in Figure 31. ${ }^{[153]}$ The reflectivity spectra of this DBR structure is also shown in the inset along with simulation results based on the transmission matrix method. The peak reflectance of $99 \%$ occurred at a center wavelength of $465 \mathrm{~nm}$ and the reflectance bandwidth is $45 \mathrm{~nm}$. Such DBRs have been fabricated for maximum reflectance from the near UV to the green part of the spectrum. Details regarding the DBRs as well as a prototype VCSEL structure have been published elsewhere. ${ }^{[142,143]}$

Our group also developed DBRs based on $30 \mathrm{AlGaN} / \mathrm{AlN}$ quarter wave stacks with maximum reflectivity of $99 \%$ at $340 \mathrm{~nm}$ by PAMBE on sapphire substrates. ${ }^{[144]}$ As expected these DBRs have a narrower reflectance bandwidth of about $20 \mathrm{~nm}$. In this work we had employed various types of buffers on the sapphire substrate to address issues associated with the tensile stress in the AIN layers, which leads to nucleation and propagation of cracks.

The development of deep UV reflecting AlGaN-based DBRs is challenging because of the smaller index of refraction contrast of the layers in the quarter wave stacks. Furthermore, the development of conducting DBRs, which are required for resonant cavity devices is even more challenging because of the difficulty in doping AlGaN alloys with high AlN mole fraction n-type and particularly p-type. One possible solution proposed by Brummer et al. in our group is to employ compositionally graded AlGaN alloys as schematically shown in Figure 32. ${ }^{[145]}$ As discussed previously in graded AlGaN alloys, free electron and hole densities are determined by electric fields from polarization charges rather than by thermal activation of dopants. Both n- and p-type doping, resulting from remote mobile carriers accumulating on fixed polarization charge, have been reported in graded composition AlGaN alloys. ${ }^{[137,154]}$ The experimental and simulation results of the reflectivity for these DBRs are shown in Figure 33. ${ }^{[145]}$

\section{Acknowledgments}

I would like to thank all of my students whose work on AlGaN alloys and devices have been reviewed in this article. Thanks are also due to a number of colleagues at Boston University and other Institutes with whom I collaborated on the topics discussed in this article (Professors Soumendra Basu, Enrico Bellotti, Luca Dal Negro, Clemens Heske, Theodore Karakostas, Philomela Komninou, Karl Ludwig, Roberto Paiella, Kevin Smith, and David Smith; Drs Emanuel Dimakis, Alexey Nikiforov, and Emanuele Pecora). This work was supported in part by the NSF Division of Electrical, Communications, and Cyber Systems standard Grant No.1408364, overseen by Drs. Mahmoud Fallahi and John Zavada.

\section{References}

1. J.I. Pankove and T.D. Moustakas, eds.: Gallium nitride I. In Semiconductors and Semimetals (Academic Press, San Diego, 50, 1998).

2. J.I. Pankove and T.D. Moustakas, eds.: Gallium nitride II. In Semiconductors and Semimetals (Academic Press, San Diego, 57, 1999).

3. S. Nakamura, S. Pearton, and G. Fasol: The Blue Laser Diode, 2nd ed. (Springer, Berlin, 2000).

4. H. Morkoc: Handbook of Nitride Semiconductors and Devices, Vol. 3: GaN-based Optical and Electronic Devices (WILEY-VCH, Weinheim, 2009).

5. A. Khan, K. Balakrishnan, and T. Katona: Ultraviolet light-emitting diodes based on group-III nitrides, Nat. Photonics 2, 77 (2008).

6. H. Yoshida, Y. Yamashita, M. Kuwabara, and H. Kan: A 342-nm ultraviolet AIGaN multiple-quantum-well laser diode. Nat. Photonics 2, 551554 (2008).

7. C. Pernot, M. Kim, S. Fukahori, T. Inazu, T. Fujita, Y. Nagasawa, A. Hirano, M. Ippommatsu, M. Iwaya, S. Kamiyama, I. Akasaki, and H. Amano: Improved efficiency of $255-280 \mathrm{~nm}$ AlGaN-based lightemitting diodes. Appl. Phys. Express 3, 061004 (2010).

8. H. Hirayama, Y. Tsukada, T. Maeda, and N. Kamata: Marked enhancement in the efficiency of deep-ultraviolet AIGaN light-emitting diodes by using a multiquantum-barrier electron blocking layer. Appl. Phys. Express 3, 031002 (2010).

9. R. Grandusky, S.R. Gibb, M.C. Mendrick, C. Moe, M. Wraback, and L.J. Schowalter: High output power from $260 \mathrm{~nm}$ pseudomorphic ultraviolet light-emitting diodes with improved thermal performance. Appl. Phys. Express 4, 082101 (2011).

10. M. Shatalov, W. Sun, A. Lunev, X. Hu, A. Dobrinsky, Y. Bilenko, J. Yang, M. Shur, R. Gaska, C. Moe, G. Garrett, and M. Wraback: AlGaN deep- 
ultraviolet light-emitting diodes with external quantum efficiency above 10\%. Appl. Phys. Express 5, 082101 (2012).

11. M.R. Krames, O.B. Shchekin, R. Mueller-Mach, G.O. Mueller, L. Zhou, G. Harbers, and M.G. Craford: Status and future of high-power light-emitting diodes for solid-state lighting. J. Display Technol. 3, 160 (2007).

12. T. Takano, Y. Narita, A. Horiuchi, and H. Kawanishi: Room-temperature deep-ultraviolet lasing at $241.5 \mathrm{~nm}$ of AIGaN multiple-quantum-well laser. Appl. Phys. Lett. 84, 3567 (2004).

13. V.N. Jmerik, A.M. Mizerov, A.A. Sitnikova, P.S. Kop'ev, S.V. Ivanov, E.V. Lutsenko, N.P. Tarasuk, N.V. Rzheutskii, and G.P. Yablonskii: Low-threshold $303 \mathrm{~nm}$ lasing in AlGaN-based multiple-quantum well structures with an asymmetric waveguide grown by plasma-assisted molecular beam epitaxy on c-sapphire. Appl. Phys. Lett. 96, 141112 (2010).

14. T. Wunderer, C.L. Chua, Z. Yang, J.E. Northrup, N.M. Johnson, G. A. Garrett, H. Shen, and M. Wraback: Pseudomorphically grown ultraviolet $\mathrm{C}$ photopumped lasers on bulk AIN substrates. Appl. Phys. Express 4, 092101 (2011).

15. J. Mickevicius, J. Jurkevicius, K. Kazlauskas, A. Zakauskas, G. Tamulaitis, M.S. Shur, M. Shatalov, J. Yang, and R. Gaska: Stimulated emission in AIGaN/AIGaN quantum wells with different $\mathrm{Al}$ content. Appl. Phys. Lett. 100, 081902 (2012).

16. E.F. Pecora, W. Zhang, A.Yu Nikiforov, L. Zhou, D.J. Smith, J. Yin, R. Paiella, L. Dal Negro, and T.D. Moustakas: Sub-250 nm roomtemperature optical gain from AIGaN/AIN multiple quantum wells with strong band-structure potential fluctuations. Appl. Phys. Lett. 100, 061111-061114 (2012).

17. Z. Lochner, T.T. Kao, Y.S. Liu, X.H. Li, M.M. Satter, S.C. Shen, P.D. Yoder, J.H. Ryou, R.D. Dupuis, Y. Wei, H. Xie, A. Fischer, and F.A. Ponce: Deep-ultraviolet lasing at $243 \mathrm{~nm}$ from photo-pumped AIGaN/AIN heterostructure on AIN substrate. Appl. Phys. Lett. 102, $101110(2013)$

18. E.F. Pecora, W. Zhang, A.Yu Nikiforov, J. Yin, R. Paiella, L. Dal Negro, and T.D. Moustakas: Sub-250 nm light emission and optical gain in AlGaN materials. J. Appl. Phys. 113, 013106 (2013).

19. V.N. Jmerik, E.V. Lutsenko, and S.V. Ivanov: Plasma-assisted molecular beam epitaxy of AIGaN heterostructures for deep-ultraviolet optically pumped lasers. Phys. Status Solidi A 210-450, 439 (2013).

20. H. Yoshida, Y. Yamashita, M. Kuwabara, and H. Kan: Demonstration of an ultraviolet $336 \mathrm{~nm}$ AIGaN multiple-quantum-well laser diode. Appl. Phys. Lett. 93, 241106 (2008).

21. M.A. Khan, J.N. Kuznia, D.T. Olson, J.M. Van Hove, and M. Blasingame: High-responsivity photoconductive ultraviolet sensors based on insulating single-crystal GaN epilayers. Appl. Phys. Lett. 60, 2917-2919 (1992).

22. M. Misra, T.D. Moustakas, R.P. Vaudo, R. Singh, and K.S. Shah: Photoconducting ultraviolet detectors based on GaN films grown by electron cyclotron resonance. Mol. Beam Epitaxy, Proc. SPIE 2519, 78-86 (1995)

23. M. Razeghi and A. Rogalski: Semiconductor ultraviolet detectors. J. Appl. Phys. 79, 7433-7473 (1996).

24. J.C. Carrano, T. Li, P.A. Grudowski, C.J. Eiting, R.D. Dupuis, and J. C. Campbell: Comprehensive characterization of metal-semiconductormetal ultraviolet photodetectors fabricated on single-crystal GaN. J. Appl. Phys. 83, 6148-6160 (1998).

25. M. Misra, D. Korakakis, H.M. Ng, and T.D. Moustakas: Photoconductive detectors based on partially ordered $\mathrm{Al}_{x} \mathrm{Ga}_{1-x} \mathrm{~N}$ alloys grown by molecular beam epitaxy. Appl. Phys. Lett. 74, 2203-2205 (1999).

26. E. Monroy, F. Calle, J.L. Pau, E. Muñoz, F. Omnès, B. Beaumont, and P. Gibart: AlGaN-based UV photodetectors. J. Cryst. Growth 230, 537-543 (2001)

27. V. Kuryatkov, A. Chandolu, B. Borisov, G. Kipshidze, K. Zhu, S. Nikishin, and $\mathrm{H}$. Temkin: Solar-blind ultraviolet photodetectors based on superlattices of AIN/AIGa(In)N. Appl. Phys. Lett. 82, 1323-1325 (2003).

28. T.D. Moustakas and M. Misra: Origin of the high photoconductive gain in AIGaN films. Proc. SPIE 6766, 67660C (2007).

29. M. Misra, A. Bhattacharyya, and T.D. Moustakas: Nitride-based UV detectors improve photodetection. Laser Focus World, pp. 64-66 (2008) (www.laserfocusworld.com).
30. M. Kneissl, T.L. Paoli, P. Kiesel, D.W. Treat, M. Teepe, N. Miyashita, and N. M. Johnson: Two-section InGaN multiple-quantum-well laser diode with integrated electroabsorption modulator. Appl. Phys. Lett. 80, 3283 (2002).

31. A.E. Oberhofer, J.F. Muth, M.A.L. Johnson, Z.Y. Chen, E.F. Fleet, and G. D. Cooper: Planar gallium nitride ultraviolet optical modulator. Appl. Phys. Lett. 83, 2748 (2003).

32. I. Friel, C. Thomidis, and T.D. Moustakas: Ultraviolet electroabsorption modulator based on AIGaN/GaN multiple quantum wells. J. Appl. Phys. 97, 123515 (2005)

33. E. Sari, S. Nizamoglu, T. Ozel, and H.V. Demir: Blue quantum electroabsorption modulators based on reversed quantum confined Stark effect with blueshift. Appl. Phys. Lett. 90, 011101 (2007).

34. T. Ozel, E. Sari, S. Nizamoglu, and H.V. Demir: Violet to deep-ultraviolet InGaN/GaN and GaN/AIGaN quantum structures for UV electroabsorption modulators. J. Appl. Phys. 102, 113101 (2007).

35. C-K. Kao, A. Bhattacharyya, C. Thomidis, R. Paiella, and T.D. Moustakas: Electroabsorption modulators based on bulk GaN films and GaN /AIGaN multiple quantum wells. J. Appl. Phys. 109, 083102 (2011).

36. C-K. Kao, A. Bhattacharyya, C. Thomidis, A. Moldawer, R. Paiella, and T. D. Moustakas: A comparative study of UV electroabsorption modulators based on bulk III-nitride films and multiple quantum wells. Phys. Status Solidi C 9, 770-773 (2012).

37. R.J. Molnar, R. Singh, and T.D. Moustakas: The effect of plasma source exit apertures on the growth of gallium nitride by the method of electron cyclotron resonance plasma-assisted molecular beam epitaxy (ECRMBE). J. Electron. Mater. 24, 275 (1995).

38. T.D. Moustakas, R.J. Molnar, G. Menon, and C.R. Eddy Jr.: A comparative study of GaN Films grown on different faces of Sapphire by ECR-assisted MBE. In Wide Band Gap Semiconductors, edited by T. D. Moustakas, J.I. Pankove, and Y. Hamakawa (Mat. Res. Soc. Symp. Proc, Pittsburgh, 242, 1992), pp. 427-432.

39. T.D. Moustakas, T. Lei, and R.J. Molnar: Growth of GaN by ECR-assisted MBE. Physica B 185, 36-49 (1993)

40. Y. Wang, A. Özcan, G. Özaydin, K. Ludwig Jr., A. Bhattacharyya, T. D. Moustakas, H. Zhou, R. Headrick, and D.P. Siddons: Real time synchrotron x-ray studies of low and high temperature nitridation of c-plane sapphire. Phys. Rev. B 74, 235304 (2006).

41. T.D. Moustakas: In Semiconductors and Semimetals, edited by J.I. Pankove and T.D. Moustakas (Academic Press, 57, New York, 1998), Chap. 2.

42. D. Doppalapudi, E. Iliopoulos, S.N. Basu, and T.D. Moustakas: Epitaxial growth of gallium nitride thin films on A-plane sapphire by molecular beam epitaxy. J. Appl. Phys. 85, 3582 (1999).

43. H. Amano, N. Sawaki, I. Akasaki, and Y. Toyoda: Metalorganic vapor phase epitaxial growth of a high quality GaN film using an AIN buffer layer. Appl. Phys. Lett. 48, 353 (1986).

44. A.N. Wright and K.A. Winkler: Active Nitrogen (Academic Press, New York, 1968).

45. T.D. Moustakas: The role of extended defects on the performance of optoelectronic devices in nitride semiconductors. Phys. Status Solidi $A$ 210, 169-174 (2013).

46. W.D. Nix and B.M. Clemens: Crystallite coalescence: a mechanism for intrinsic tensile stresses in thin films. J. Mater. Res. 14, 3467 (1999).

47. T.D. Moustakas, Y. Liao, C-K. Kao, C. Thomidis, A. Bhattacharyya D. Bhattarai, and A. Moldawer: Deep UV-LEDs with high IQE based on AIGaN alloys with strong band structure potential fluctuations. Proc. SPIE 8278, 82780L (2012).

48. C.R. Elsass, T. Mates, B. Heying, C. Poblenz, P. Fini, P.M. Petroff, S. P. DenBaars, and J.S. Speck: Effects of growth conditions on the incorporation of oxygen in AlGaN layers grown by plasma assisted molecular beam epitaxy. Appl. Phys. Lett. 77, 3167 (2000).

49. T.D. Moustakas and A. Bhattacharyya: Experimental evidence that the plasma-assisted MBE growth of nitride alloys is a liquid phase Epitaxy process. ECS Trans. 35, 63-71 (2011).

50. T.D. Moustakas and A. Bhattacharyya: The role of liquid phase epitaxy during growth of AIGaN by MBE. Phys. Status Solidi C 9, 580-583 (2012).

51. M.D. McCluskey, N.M. Johnson, C.G. Van de Walle, D.B. Bour, M. Kneissl, and W. Walukiewicz: Metastability of oxygen donors in AlGaN. Phys. Rev. Lett. 80, 4008 (1998). 
52. Y. Taniyasu, M. Kasu, and T. Makimoto: An aluminium nitride lightemitting diode with a wavelength of 210 nanometres. Nature 441, 325 (2006).

53. K.B. Nam, J. Li, M.L. Nakarmi, J.Y. Lin, and H.X. Jiang: Unique optical properties of AlGaN alloys and related ultraviolet emitters. Appl. Phys. Lett. 84, 5264 (2004).

54. E. Monroy, B. Daudin, E. Bellet-Amalric, N. Gogneau, D. Jalabert, J. Brault, J. Barjon, and Le Si Dang: Surfactant effect of In for AIGaN growth by plasma-assisted molecular beam epitaxy. J. Appl. Phys. 93, 1550 (2003).

55. T.D. Moustakas and R.J. Molnar: Growth and doping of GaN films by ECR-assisted MBE. Materials Research Society Symp. Proc. Volume 281, 1993, p. 753.

56. B. Heying, R. Averbeck, L.F. Chen, E. Haus, H. Riechert, and J.S. Speck: Control of GaN surface morphologies using plasma-assisted molecular beam epitaxy. J. Appl. Phys. 88, 1855-1860 (2000).

57. G. Mula, C. Adelmann, S. Moehl, J. Oullier, and B. Daudin: Surfactant effect of gallium during molecular-beam epitaxy of GaN on AIN (0001). Phys. Rev. B 64, 195406 (2001).

58. T. Zywietz, J. Neugebauer, and M. Scheffler: Adatom diffusion at GaN (0001) and (0001) surfaces. Appl. Phys. Lett. 73, 487 (1998).

59. J.E. Northrup, J. Neugebauer, R.M. Feenstra, and A.R. Smith: Structure of GaN (0001): the laterally contracted Ga bilayer model. Phys. Rev. B 61, 9932-9935 (2000).

60. J. Neugebauer, T.K. Zywietz, M. Scheffler, J.E. Northrup, H. Chen and R. M. Feenstra: Adatom kinetics on and below the surface: the existence of a new diffusion channel. Phys. Rev. Lett. 90, 56101 (2003).

61. T.D. Moustakas and A. Bhattacharyya: 24th NAMBE, Duke University, Oct. 9, 2006, paper no. MB-8 (unpublished); T.D. Moustakas and A. Bhattacharyya: $7^{\text {th }}$ ICNS, Las Vegas, Sept. 16, 2007, Abstracts p. 112.

62. T.D. Moustakas and A. Bhattacharyya: Experimental evidence that the plasma-assisted MBE growth of nitride alloys is a liquid phase epitaxy process. ECS Trans. 35, 63-71 (2011).

63. J. Karpinski, J. Jun, and S. Porowski: Equilibrium pressure of $\mathrm{N}_{2}$ over GaN and high pressure solution growth of GaN. J. Cryst. Growth $\mathbf{6 6}$, 1-10 (1984).

64. D. Elwell, R.S. Feigelson, M.M. Simkins, and W.A. Tiller: Crystal growth of GaN by the reaction between gallium and ammonia. J. Cryst. Growth 66, 45-54 (1984).

65. A. Argoitia, C.C. Hayman, and J.C. Angus: Low pressure synthesis of bulk, polycrystalline gallium nitride. Appl. Phys. Lett. 70, 179-181 (1997).

66. T.D. Moustakas and J.P. Dismukes: Growth of bulk GaN by reaction of $\mathrm{Ga} / \mathrm{Sn}$ with activated nitrogen. In III-V Nitride Material and Processes, edited by C.R. Abernathy et al. (ECS Proc., 284, 1998), pp. 97-34.

67. C.W. Hu, A. Bell, F.A. Ponce, D.J. Smith, and S.T. Tsong: Growth of selfassembled GaN quantum dots via the vapor-liquid-solid mechanism. Appl. Phys. Lett. 81, 3236-3238 (2002).

68. Y. Wang, A. Ozcan, C. Sanborn, K. Ludwig, A. Bhattacharyya, R. Chandrasekaran, T.D. Moustakas, L. Zhou, and D. Smith: Real-time $x$-ray studies of gallium nitride nanodot formation by droplet heteroepitaxy. J. Appl. Phys. 102, 073522 (2007).

69. E. Iliopoulos and T.D. Moustakas: Growth kinetics of AIGaN films by plasma assisted molecular beam epitaxy. Appl. Phys. Lett. 81, 295 (2002).

70. T.D. Moustakas: Molecular beam epitaxy: thin film growth and surface studies. MRS Bull. XIII, 29-34 (1988).

71. A. Bhattacharyya, T.D. Moustakas, L. Zhou, D.J. Smith, and W. Hug: Deep ultraviolet emitting AIGaN quantum wells with high internal quantum efficiency. Appl. Phys. Lett. 94, 181907 (2009).

72. T.P. Pearsall, ed.: GalnAsP Alloy Semiconductors (Wiley, New York, 1982).

73. W.E. Hoke, A. Torabi, J.J. Mosca, and T.D. Kenedy: Thermodynamic analysis of cation incorporation during molecular beam epitaxy of nitride films using metal-rich growth conditions. J. Vac. Sci. Technol. B 25, 978-982 (2007).

74. A. Zunger, and S. Mahajan: Atomic ordering and phase separation in III-V alloy semiconductors. In Handbook of Semiconductors, 2nd ed., edited by T.S. Moss (Elsevier, 3, Amsterdam, 1994).
75. A. Gomyo, T. Suzuki, and S. lijima: Observation of strong ordering in $\mathrm{Ga}_{x} \mathrm{In}_{1-x} \mathrm{P}$ alloy semiconductors. Phys. Rev. Lett. 60, 2645 (1988).

76. L.C. Su, I.H. Ho, and G.B. Stringfellow: Kinetically controlled order/disorder structure in GalnP. Appl. Phys. Lett. 65, 749 (1994).

77. R. Osorio, J.E. Bernard, S. Froyen, and A. Zunger: Ordering thermodynamics of surface and subsurface layers in the $\mathrm{Ga}_{1-x} \ln _{\mathrm{x}} \mathrm{P}$ alloy. Phys. Rev. B 45, 11173 (1992).

78. D.J. Friedman, J.G. Zhu, A.E. Kibbler, J.M. Olson, and J. Moreland: Surface topography and ordering-variant segregation in $\mathrm{GalnP}_{2}$. Appl. Phys. Lett. 63, 1774 (1993).

79. D. Korakakis, K.F. Ludwig, and T.D. Moustakas: Long range order in $\mathrm{Al}_{x} \mathrm{Ga}_{1-x} \mathrm{~N}$ films grown by ECR-assisted MBE. Appl. Phys. Lett. 71, 72 (1997).

80. E. Iliopoulos, K.F. Ludwig Jr., and T.D. Moustakas: Complex ordering in ternary wurtzite nitride alloys. J. Phys. Chem. Solids 64, 1525 (2003).

81. J.C. Woicik, K.F. Ludwig Jr., and T.D. Moustakas: Composition dependent bilayer atomic ordering in AlGaN films examined by polarizationdependent extended X-ray absorption fine structure. Appl. Phys. Lett. 100, 162105 (2012)

82. J.E. Northrup, L.T. Romano, and J. Neugebauer: Surface energetics, pit formation, and chemical ordering in InGaN alloys. Appl. Phys. Lett. 74, 2319 (1999)

83. E. Iliopoulos, K.F. Ludwig, T.D. Moustakas, and S.N.G. Chu: Chemical ordering in AIGaN alloys grown by molecular beam epitaxy. Appl. Phys. Lett. 78, 463 (2001).

84. E. Iliopoulos, K.F. Ludwig Jr., T.D. Moustakas, Ph. Komninou, Th. Karakostas, G. Nouet, S.N.G. Chu: Epitaxial growth and selforganized superlattice structures in AlGaN films grown by plasma assisted molecular beam epitaxy. Mater. Sci. Eng. B 87, 227 (2001).

85. Y. Wang, A.S. Ozcan, K.F. Ludwig Jr., A. Bhattacharyya, T.D. Moustakas, L. Zhou, and D. Smith: Complex and incommensurate ordering in $\mathrm{Al}_{0.72}$ $\mathrm{Ga}_{0.28} \mathrm{~N}$ thin films grown by plasma assisted molecular beam epitaxy. Appl. Phys. Lett. 88, 181915 (2006).

86. M. Behbehani, E. Piner, S. Liu, N. El-Masry, and S. Bedair: Phase separation and ordering coexisting in $\mathrm{In}_{\mathrm{x}} \mathrm{Ga}_{1-\mathrm{x}} \mathrm{N}$ grown by metal organicchemical vapor deposition. Appl. Phys. Lett. 75, 2202 (1999).

87. P. Ruterana, G. De Saint Jores, M. Laugt, F. Omnes, and E. BelletAmalric: Evidence for multiple chemical ordering in AlGaN grown by metalorganic chemical vapor deposition. Appl. Phys. Lett. 78, 344 (2001).

88. D. Doppalapudi, S.N. Basu, and T.D. Moustakas: Domain structure in chemically ordered $\operatorname{In}_{x} \mathrm{Ga}_{1-x} \mathrm{~N}$ alloys grown by molecular beam epitaxy. J. Appl. Phys. 85, 883 (1999).

89. E. Iliopoulos: Ph.D. Dissertation (Boston University, 2002).

90. H.G. Lee, M. Gershenzon, and B.L. Goldenberg: Ultraviolet photoluminescence from undoped and $\mathrm{Zn}$ doped $\mathrm{Al}_{x} \mathrm{Ga}_{1_{-} \mathrm{N}} \mathrm{N}$ with $\mathrm{x}$ between 0 and 0.75. J. Electron. Mater. 20, 621 (1991).

91. M.D. McCluskey, N.M. Johnson, C.G. Van de Walle, D.P. Bour, and M. Kneissl: Metastability of oxygen donors in AlGaN. Phys. Rev. Lett. 80, 4008 (1998).

92. M.D. Bremser, W.G. Perry, T. Zheleva, N.V. Edwards, O.H. Nam, N. Parikh, E.E. Aspnes, and R.F. Davis: Growth, doping and characterization of $\mathrm{Al}_{x} \mathrm{Ga}_{1-x} \mathrm{~N}$ thin film alloys on $6 \mathrm{H}-\mathrm{SiC}$ (0001) substrates MRS internet. J. Nitride Semicond. Res. 1, 8 (1996).

93. Y. Taniyasu, M. Kasu, and N. Kobayashi: Intentional control of n-type conduction for Si-doped AIN and $\mathrm{Al}_{x} \mathrm{Ga}_{1-\mathrm{x}} \mathrm{N}(0.42<\mathrm{x}<1)$. Appl. Phys. Lett. 81, 1255 (2002)

94. C. Skierbiszewski, T. Suski, and M. Leszczynski: Evidence for localized Si-donor state and its metastable properties in AlGaN. Appl. Phys. Lett. 74, 3833 (1999).

95. C. Stampfl and C.G. Van de Walle: Doping of $\mathrm{Al}_{\mathrm{x}} \mathrm{Ga}_{1-\mathrm{x}}$ N. Appl. Phys. Lett. 72, 459 (1998).

96. J. Hwang, W.J. Schaff, L.F. Eastman, S.T. Bradley, L.J. Brillson, D. C. Look, J. Wu, W. Walukiewicz, M. Furis, and A.N. Cartwright: Si doping of high-Al-mole fraction $\mathrm{Al}_{x} \mathrm{Ga}_{1-x} \mathrm{~N}$ alloys with if plasma-induced molecular-beam-epitaxy. Appl. Phys. Lett. 81, 5192 (2002).

97. S.T. Bradley, S.H. Goss, and L.J. Brillson: Deep level defects and doping in high Al mole fraction AlGaN. J. Vac. Sci. Technol. B 21, 2558 (2003). 
98. M. Hermann, F. Futmayr, A. Bargmaier, G. Dollinger, M. Stutzmann, and M. Eickoff: Highly Si-doped AIN grown by plasma-assisted molecularbeam epitaxy. Appl. Phys. Lett. 86, 192108 (2005).

99. T. Xu., C. Thomidis, I. Friel, and T.D. Moustakas: Growth and silicon doping of AlGaN films in the entire alloy composition by molecular beam epitaxy. Phys. Status Solidi C 2(7), 2220 (2005).

100.A. Bhattacharyya, W. Li, J. Cabalu, T.D. Moustakas, D.J. Smith, and R. L. Hervig: Efficient p-type doping of GaN films by plasma-assisted molecular beam epitaxy. Appl. Phys. Lett. 85, 4956 (2004).

101.W. Li: Ph.D. Dissertation (Boston University, 2008).

102.I. Suemune: Doping in a superlattice structure: improved hole activation in widegap II-VI materials. J. Appl. Phys. 67, 2364 (1990).

103.P. Kozodoy, M. Hansen, S.P. DenBaars, and U.K. Mishra: Enhanced Mg doping efficiency in $\mathrm{Al}_{0.2} \mathrm{Ga}_{0.8} \mathrm{~N} / \mathrm{GaN}$ superlattices. Appl. Phys. Lett. 74, 3681 (1999).

104.A. Saxler, W.C. Mitchel, P. Kung, and M. Razeghi: Aluminum gallium nitride short-period superlattices doped with magnesium. Appl. Phys. Lett. 74, 2023 (1999).

105.I.D. Goepfert, E.F. Schubert, A. Osinsky, P.E. Norris, and N.N. Faleev: Experimental and theoretical study of acceptor activation and transport properties in p-type $\mathrm{Al}_{x} \mathrm{Ga}_{1-x} \mathrm{~N} / \mathrm{GaN}$ superlattices. J. Appl. Phys. 88, 2030 (2000).

106.S.F. Chichibu, A. Uedono, T. Onuma, B.A. Haskell, A. Chakraborty, T. Koyama, P.T. Fini, S. Keller, S.P. Denbaars, J.S. Speck, U. K. Mishra, S. Nakamura, S. Yamaguchi, S. Kamiyama, H. Amano, I. Akasaki, J. Han, and T. Sota: Origin of defect-insensitive emission probability in In-containing (Al,In,Ga)N alloy semiconductors. Nat. Mater. 5, 810-816 (2006).

107.W. Zhang, A.Yu Nikiforov, C. Thomidis, J. Woodward, H. Sun, C-K. Kao, D. Bhattarai, A. Moldawer, L. Zhou, D.J. Smith, and T.D. Moustakas: MBE growth of AIGaN quantum wells on $6 \mathrm{H}-\mathrm{SiC}$ substrates with high internal quantum efficiency. J. Vac. Sci. Technol. B 30, 02B119 (2012).

108.D. Doppalapudi and T.D. Moustakas: Epitaxial growth and structure of III-V nitride thin films. In Handbook of Thin Film Materials, edited by H.S. Nalwa (Academic Press, San Diego, 4, 2002), Chap. 2.

109.J.C. Cabalu, A. Bhattacharyya, C. Thomidis, T.D. Moustakas, and C. J. Collins: High power ultraviolet light emitting diodes based on GaN/ AIGaN quantum wells produced by molecular beam epitaxy. J. Appl. Phys. 100, 104506 (2006).

110.J.C. Cabalu, C. Thomidis, I. Friel and T.D. Moustakas, and S. Riyopoulos: Enhanced internal quantum efficiency and light extraction efficiency from textured GaN/AIGaN quantum wells grown by molecular beam epitaxy. J. Appl. Phys. 99, 064904 (2006).

111.Y. Liao, C. Thomidis, C-K. Kao, A. Moldawer, W. Zhang, Y.-C. Chang, A. Yu Nikiforov, E. Bellotti, and T.D. Moustakas: Milliwatt power AlGaN-based deep ultraviolet LEDs by plasma-assisted MBE. Phys. Status Solidi Rapid Res. Lett. 4, 49-51 (2010).

112.Y. Liao, C. Thomidis, A. Bhattacharyya, C-K. Kao, A. Moldawer, W. Zhang, and T.D. Moustakas: Development of milliwatt power AlGaN-based deep UV-LEDs by Plasma-assisted MBE. Materials Research Society Symp. Proc. Volume 1202, paper number 1202-I10-01, 2010.

113. Y. Liao, C. Thomidis, C-K. Kao, and T.D. Moustakas: AlGaN based deep ultraviolet light emitting diodes with high internal quantum efficiency grown by molecular beam epitaxy. Appl. Phys. Lett. 98, 081110 (2011).

114. Y. Liao, C-K. Kao, C. Thomidis, A. Moldawer, J. Woodward, D. Bhattarai, and T.D. Moustakas: Recent progress of efficient deep UV-LEDs by plasma-assisted molecular beam epitaxy. Phys. Status Solidi C $\mathbf{9}$, 798-801 (2012)

115. L.T. Romano, C.G. Van de Walle, J.W. Ager III, W. Gotz, and R.S. Kern: Effect of Si doping on strain, cracking, and microstructure in GaN thin films grown by metalorganic chemical vapor deposition. J. Appl. Phys. 87, 7745 (2000).

116.J.P. Zhang, H.M. Wang, M.E. Gaevski, C.Q. Chen, Q. Fareed, J.W. Yang, G. Simin, and M. Asif Khan: Crack-free thick AIGaN grown on sapphire using AIN/AIGaN superlattice for strain management. Appl. Phys. Lett. 80, 3542-3544 (2002).

117.K. Mayes, A. Yasan, R. McClintock, D. Shiell, S.R. Darvish, P. Kung, and M. Razeghi: Effect of Si doping on strain, cracking, and microstructure in
GaN thin films grown by metalorganic chemical vapor deposition. Appl. Phys. Lett. 84, 1046 (2004).

118.R. France, T. Xu, P. Chen, R. Chandrasekaran, and T.D. Moustakas: Vanadium-based Ohmic contacts to n-AlGaN in the entire alloy composition. Appl. Phys. Lett. 90, 062115 (2007).

119.S. Pookpanratana, R. France, M. Bar, L. Weihardt, O. Fuchs, M. Blum, W. Yang, J.D. Denlinger, T.D. Moustakas, and C. Heske: Intermixing and chemical structure at the interface between n-GaN and V-based contacts. Appl. Phys. Lett. 93, 172106 (2008).

120.S. Pookpanratana, R. France, M. Blum, A. Bell, M. Bar, L. Weinhardt, Y. Zhang, T. Hofmann, O. Fuchs, W. Yang, J.D. Denlinger, S. Mulcahy, T.D. Moustakas, and C. Heske: Chemical structure of vanadium-based contact formation on n-AIN. J. Appl. Phys. 108, 24906 (2010).

121.S. Pookpanratana, R. France, R. Félix, R. Wilks, L. Weinhardt, T. Hofmann, L. Tati Bismaths, S. Mulcahy, F. Kronast, T.D. Moustakas, M. Bär, and C. Heske: Microstructure of vanadium-based contacts on n-GaN. J. Phys. D: Appl. Phys. 45, 105401 (2012).

122.S.L. Chuang: Optical gain of strained wurtzite GaN quantum-well lasers. IEEE J. Quantum Electron. 32, 1791 (1996).

123.E.F. Pecora, W. Zhang, J. Yin, R. Paiella, L. Dal Negro, and T. D. Moustakas: Polarization properties of deep-UV optical gain in Al-rich AlGaN structures. Appl. Phys. Express 5, 032103 (2012).

124.K.L. Shaklee, R.E. Nahory, and R.F. Leheny: Optical gain in semiconductors. J. Lumin. 7, 284-309 (1973)

125.L. Dal Negro, M. Cazzanelli, L. Pavesi, S. Ossicini, D. Pacifici, G. Franzo, F. Priolo, and F. lacona: Dynamics of stimulated emission in silicon nanocrystals. Appl. Phys. Lett. 82, 4636 (2003).

126.L. Dal Negro, P. Bettotti, M. Cazzanelli, D. Pacifici, and L. Pavesi: Applicability conditions and experimental analysis of the variable stripe length method for gain measurements. Opt. Commun. 229, 337-348 (2004).

127.G. Frankowsky, F. Steuber, V. Harle, F. Scholz, and A. Hangleiter: Optical gain in GalnN/GaN heterostructures. Appl. Phys. Lett. 68, 3746 (1996).

128.M. Röwe, M. Vehse, P. Michler, J. Gutowski, S. Heppel, and A. Hangleiter: Optical gain, gain saturation, and waveguiding in group III-nitride heterostructures. Phys. Status Solidi C 0, 1860-1877 (2003).

129.H. Sun, E.F. Pecora, J. Woodward, D.J. Smith, L. Dal Negro, and T. D. Moustakas: Effect of Indium in $\mathrm{Al}_{0.65} \mathrm{Ga}_{0.35} \mathrm{~N} / \mathrm{Al}_{0.8} \mathrm{Ga}_{0.2} \mathrm{~N}$ MQWs for the development of deep UV laser structures in the form of graded-index separate confinement heterostructure (GRINSCH). Phys. Status Solidi A 213, 1165-1169 (2016).

130.E. Northrup, C.L. Chua, Z. Yang, T. Wunderer, M. KneissI, N.M. Johnson, and T. Kolbe: Effect of strain and barrier composition on the polarization of light emission from AIGaN/AIN quantum wells. Appl. Phys. Lett. 100, 021101 (2012).

131.J. Zhang, H. Zhao, and N. Tansu: Effect of crystal-field split-off hole and heavy-hole bands crossover on gain characteristics of high Al-content AlGaN quantum well lasers. Appl. Phys. Lett. 97, 111105 (2010).

132.A.A. Yamaguchi: Theoretical investigation of optical polarization properties in Al-rich AlGaN quantum wells with various substrate orientations. Appl. Phys. Lett. 96, 151911 (2010).

133.S. Park: Al composition dependence of the optical gain characteristics of a-plane Al-rich AlGaN/AIN quantum-well structures. J. Korean Phys. Soc. 59, 357 (2011)

134.H. Sun, J. Woodward, J. Yin, A. Moldawer, E.F. Pecora, A.Yu Nikiforov, L. Dal Negro, R. Paiella, K. Ludwig Jr., D.J. Smith, and T.D. Moustakas: Development of AIGaN-based GRINSCH deep UV emitters by molecular beam epitaxy. J. Vac. Sci. Technol. B 31, 03 C117 (2013).

135.H. Sun and T.D. Moustakas: UV emitters based on an AIGaN p-n junction in the form of graded-index separate confinement heterostructure. Appl. Phys. Express 7, 012104 (2014).

136.E.F. Pecora, H. Sun, L. Dal Negro, and T.D. Moustakas: Deep-UV optical gain in AlGaN-based graded-index separate confinement heterostructure. Opt. Mater. Express 5, 809-817 (2015).

137.D. Jena, S. Heikman, D. Green, D. Buttari, R. Coffie, H. Xing, S. Keller, S. DenBaars, J.S. Speck, U.K. Mishra, and I. Smorchkova: Realization of wide electron slabs by polarization bulk doping in graded III-V nitride semiconductor alloys. Appl. Phys. Lett. 81, 4395 (2002). 
138. M. Misra and T.D. Moustakas: Photoconductivity recombination kinetics in GaN films. Mater. Res. Soc. Proc. 622, T5.4.1 (2000).

139.S.V. Dudiy and A. Zunger: Type I to type II transition at the interface between random and ordered domains of $\mathrm{Al}_{x} \mathrm{Ga}_{1} \grave{A}_{x} \mathrm{~N}$ alloys. Appl. Phys. Lett. 84, 1874-1876 (2004).

140.D.S. Chemla, D.A.B. Miller, and P.W. Smith: In Semiconductors and Semimetals, edited by R. Dingle (Academic, 34, New York, 1987), Chap. 5.

141.H.M. Ng, D. Doppalapudi, E. Iliopoulos, and T.D. Moustakas: Distributed Bragg reflectors based on AIN/GaN multilayers. Appl. Phys. Lett. 74, 1036 (1999).

142.H.M. Ng, T.D. Moustakas, and S.N.G. Chu: High reflectivity and broad bandwidth AIN/GaN distributed Bragg reflectors grown by molecular beam epitaxy. Appl. Phys. Lett. 76, 2818 (2000).

143.H.M. Ng and T.D. Moustakas: Group III nitride VCSELS structures grown by molecular beam epitaxy. In Physics and Simulation of Optoelectronic Devices - Proc. SPIE, Volume 3944, 2000, p. 22.

144.A. Bhattacharyya, I. Friel, S. Iyer, E. Iliopoulos, A.V. Sampath, J. Cabalu, and T.D. Moustakas: High reflectivity and crack-free AIGaN/AIN UV distributed Bragg reflectors. J. Vac. Sci. Technol. B 20, 1229 (2002).

145.G. Brummer, D. Nothern, A.Yu Nikiforov, and T.D. Moustakas: Deep UV distributed Bragg reflectors based on graded composition AIGaN alloys. Appl. Phys. Lett. 106, 221107 (2015).

146. R.-H. Yan, R. Simes, and L. Coldren: Surface-normal electroabsorption reflection modulators using Asvmrnetric Fabry-Perot structures. IEEE J. Quantum Electron. 27, 1922 (1991).

147.T. Honda, A. Katsube, T. Sakaguchi, F. Koyama, and K. Iga: Threshold estimation of GaN-based surface emitting lasers operating in ultraviolet spectral region. Jpn. J. Appl. Phys. 34, 3527 (1995).

148.D. Doppalapudi, S.N. Basu, K.F. Ludwig, and T.D. Moustakas: Phase separation and ordering in InGaN alloys grown by molecular beam epitaxy. J. Appl. Phys. 84, 1389 (1998).

149.M. Asif Khan, J.N. Kuznia, J.M. Van Hove, and D.T. Olson: Reflective filters based on single-crystal $\mathrm{GaN}_{\mathrm{Al}} \mathrm{Al}_{\mathrm{x}} \mathrm{Ga}_{1-\mathrm{x}} \mathrm{N}$ multilayers deposited using low-pressure metalorganic chemical vapor deposition. Appl. Phys. Lett. 59, 1449 (1991).

150.T. Someya and Y. Arakawa: Highly reflective GaN/Al ${ }_{0.34} \mathrm{Ga}_{0.66} \mathrm{~N}$ quarterwave reflectors grown by metal organic chemical vapor deposition. Appl. Phys. Lett. 73, 3653 (1998).

151.R. Langer, A. Barski, J. Simon, N.T. Pelekanos, O. Konovalov, R. Andre, and L.S. Dang: High-reflectivity GaN/GaAIN Bragg mirrors at blue/green wavelengths grown by molecular beam epitaxy. Appl. Phys. Lett. 74, 3610 (1999).

152.I.J. Fritz and T.J. Drummond: AIN-GaN quarter-wave reflector stack grown by gas-source MBE on (100) GaAs. Electron. Lett. 31, 68 (1995).

153.T.D. Moustakas, E. Iliopoulos, A.V. Sampath, H.M. Ng, D. Doppalapudi, M. Misra, D. Korakakis, and R. Singh: Growth and device applications of III-nitrides by MBE. J. Cryst. Growth 227-228, 13 (2001).

154.J. Simon, V. Protasenko, C. Lian, H. Xing, and D. Jena: Polarizationinduced hole doping in wide-band-gap uniaxial semiconductor heterostructures. Science 327, 60 (2010). 